\title{
氮添加对北京东灵山地区灌丛土壤呼吸的影响
}

\author{
张建华 ${ }^{1,2}$ 唐志尧 ${ }^{3}$ 沈海花 ${ }^{2}$ 方精云 2,3
}

${ }^{1}$ 忻州师范学院, 山西忻州 $034000 ;{ }^{2}$ 中国科学院植物研究所植被与环境变化国家重点实验室, 北京 100093 ; 北京大学城市与环境学院, 北京大学地表 过程分析与模拟教育部重点实验室, 北京 100871

摘 要 土壤呼吸是陆地生态系统碳收支的重要组成部分。与森林相比, 自然或半自然的灌从主要分布在养分贫㾑的地区, 通常认为它们对环境变化较为敏感。外源氮输入可能会显著影响灌从的土壤呼吸。迄今为止, 人们对大气氮沉降对灌从土壤 呼吸的影响知之甚少。该文通过氮添加试验, 研究了北京东灵山荆条(Vitex negundo var. heterophylla)和绣线菊(Spiraea salicifolia)灌从土壤呼吸及其对不同氮添加水平(对照(0)、低氮 $\left(20 \mathrm{~kg} \mathrm{~N} \cdot \mathrm{hm}^{-2} \cdot \mathrm{a}^{-1}\right)$ 、中氮 $\left(50 \mathrm{~kg} \mathrm{~N} \cdot \mathrm{hm}{ }^{-2} \cdot \mathrm{a}^{-1}\right)$ 和高氮(100 kg $\left.\mathrm{N} \cdot \mathrm{hm}^{-2} \cdot \mathrm{a}^{-1}\right)$ )的响应。结果表明: 自然条件下, 荆条和绣线菊灌从的土壤总呼吸年通量为 5.91 和 $4.23 \mathrm{t} \mathrm{C} \cdot \mathrm{hm}^{-2} \cdot \mathrm{a}^{-1}$, 异养呼吸通 量为5.76和 $3.53 \mathrm{t} \mathrm{C} \cdot \mathrm{hm}^{-2} \cdot \mathrm{a}^{-1}$, 荆条和绣线菊灌从的总呼吸和异养呼吸均与土壤温度呈显著的指数关系。荆条和绣线菊灌从土 壤总呼吸温度敏感性系数 $\left(Q_{10}\right)$ 的变化范围分别为1.44-1.58和1.43-1.98, 异养呼吸 $Q_{10}$ 的变化范围分别为 1.38-2.11和 1.49-1.88。短期氮添加抑制了荆条灌从的自养呼吸, 而对土壤总呼吸和异养呼吸影响不明显; 氮添加促进了绣线菊灌从的异 养呼吸, 而对土壤总呼吸和自养呼吸均无显著影响; 氮添加对两种灌从土壤呼吸年通量及土壤总呼吸 $Q_{10}$ 均无显著影响。

关键词 氮沉降; 土壤呼吸; 碳循环; 温度敏感性; 温带灌从

引用格式: 张建华, 唐志尧, 沈海花, 方精云 (2017). 氮添加对北京东灵山地区灌从土壤呼吸的影响. 植物生态学报, 41, 81-94. doi: 10.17521/cjpe.2016.0085

\section{Effects of nitrogen addition on soil respiration in shrublands in Mt. Dongling, Beijing, China}

ZHANG Jian-Hua ${ }^{1,2 *}$, TANG Zhi-Yao ${ }^{3}$, SHEN Hai-Hua², and FANG Jing-Yun ${ }^{2,3}$

${ }^{1}$ Xinzhou Normal University, Xinzhou, Shanxi 034000, China; ${ }^{2}$ State Key Laboratory of Vegetation and Environmental Change, Institute of Botany, Chinese Academy of Sciences, Beijing 100093, China; and ${ }^{3}$ College of Urban and Environmental Sciences, and Key Laboratory for Earth Surface Processes of the Ministry of Education, Peking University, Beijing 100871, China

\section{Abstract}

Aims Soil respiration from terrestrial ecosystems is an important component of terrestrial carbon budgets. Compared to forests, natural or semi-natural shrublands are mostly distributed in nutrient-poor sites, and usually considered to be relatively vulnerable to environmental changes. Increased nitrogen $(\mathrm{N})$ input to ecosystems may remarkably influence soil respiration in shrublands. So far the effects of $\mathrm{N}$ deposition on shrubland soil respiration are poorly understood. The aim of this study is to investigate the soil respiration of Vitex negundo var. heterophylla and Spiraea salicifolia shrublands and their response to $\mathrm{N}$ deposition.

Methods We carried out a N enrichment experiment in V. negundo var. heterophylla and S. salicifolia shrublands in Mt. Dongling, Beijing, with four $\mathrm{N}$ addition levels $\left(\mathrm{N}_{0}\right.$, control, $0 ; \mathrm{N}_{1}$, low $\mathrm{N}, 20 \mathrm{~kg} \mathrm{~N} \cdot \mathrm{hm}^{-2} \cdot \mathrm{a}^{-1} ; \mathrm{N}_{2}$, medium N, $50 \mathrm{~kg} \mathrm{~N} \cdot \mathrm{hm}^{-2} \cdot \mathrm{a}^{-1}$ and $\mathrm{N}_{3}$, high $\mathrm{N}, 100 \mathrm{~kg} \mathrm{~N} \cdot \mathrm{hm}^{-2} \cdot \mathrm{a}^{-1}$ ). Respiration was measured from 2012-2013 within all treatments.

Important findings Under natural conditions, annual total and heterotrophic respiration were 5.91 and 4.23, 5.76 and $3.53 \mathrm{t} \mathrm{C} \cdot \mathrm{hm}^{-2} \cdot \mathrm{a}^{-1}$ for the V. negundo var. heterophylla and S. salicifolia shrublands, respectively and both were not affected by short-term $\mathrm{N}$ addition. In both shrubland types, soil respiration rate exhibited significant exponential relationships with soil temperature. Temperature sensitivity $\left(Q_{10}\right)$ of total soil respiration in $V$. negundo var. heterophylla and S. salicifolia shrublands ranged from 1.44 to 1.58 and 1.43 to 1.98 , and $Q_{10}$ of heterotrophic soil respiration ranged from 1.38 to 2.11 and 1.49 to 1.88 , respectively. Short-term N addition decreased only autotrophic respiration rate during the growing season, but had no significant effects on total and heterotrophic soil respiration in $V$. negundo var. heterophylla shrubland. In contrast, $\mathrm{N}$ addition enhanced the heterotrophic soil

收稿日期Received: 2016-03-09 接受日期Accepted: 2016-09-21

*E-mail: 1042584932@qq.com; wj123-2007@163.com 
respiration rate and did not influence autotrophic and total soil respiration in S. salicifolia shrubland.

Key words nitrogen deposition; soil respiration; carbon cycle; temperature sensitivity; temperate shrublands

Citation: Zhang JH, Tang ZY, Shen HH, Fang JY (2017). Effects of nitrogen addition on soil respiration in shrublands in Mt. Dongling, Beijing, China. Chinese Journal of Plant Ecology, 41, 81-94. doi: 10.17521/cjpe.2016.0085

土壤呼吸是陆地生态系统碳循环的重要过程, 也是土壤碳库向大气输出碳的主要途径和大气 $\mathrm{CO}_{2}$ 的重要来源(刘绍辉和方精云, 1997)。全球土壤呼吸 年通量为75-120 Pg C, 是化石燃料燃烧排放量的10 倍以上(Raich \& Polter, 1995), 因此土壤呼吸的强弱 在很大程度上决定了全球气候变化与碳循环间的反 馈关系(杨庆朋等, 2011; 吴迪等, 2015)。在全球变化 背景下, 土壤呼吸速率的微小变化就可能改变大气 中 $\mathrm{CO}_{2}$ 浓度和土壤碳素的周转速率(Bronson et al., 2008; 姚辉等, 2015), 进而延缓或加剧气候变化。土 壤呼吸是一个复杂的生物化学过程, 在不同的生态 系统中土壤呼吸受众多因素的综合影响(Bowden et al., 1993; Sitaula et al., 1995; Burton et al., 1998; Fisk \& Fahey, 2001; Savage \& Davidson, 2001; Hibbard et al., 2005; 张东秋等, 2005; Davidson \& Janssens, 2006; Chen et al., 2010; 全权等, 2015)。

近几十年来, 人类活动排放到大气中的活性氮 迅速增加, 其总量超过了所有陆地生态系统自然产 生的活性氮排放, 这种趋势在未来的数十年内还将 持续下去(Galloway et al., 2004; 2008)。氮沉降的持 续增加已经对各生态系统的特征和过程产生了很大 影响(Rabalais, 2002)。大气氮沉降引起森林的土壤 酸化、影响树木生长及生物多样性, 甚至严重威胁 森林生态系统的结构与功能(Matson et al., 1999; 李 德军等, 2003; 莫江明等, 2005; 李秋玲等, 2013)。同 样, 氮沉降会降低草原物种多样性(Stevens et al., 2004)、净氮矿化(Liu et al., 2015)及活性有机碳含量 (郑娇娇等, 2012)。作为全球碳循环流通的一个关键 环节, 土壤呼吸也受到大气氮沉降增加的影响, 有 关氮沉降对土壤呼吸影响的研究多集中于森林和草 地(Han et al., 2012; 李伟斌等, 2014; Qi et al., 2014; 吴迪等, 2015)。但在不同生态系统，土壤呼吸对氮 沉降的响应并不一致, 例如, 邓琦等(2009)发现高 氮处理对鼎湖山南亚热带人工森林生态系统土壤呼 吸的影响与季节的降雨量紧密相关。不同研究发现 氮沉降可能促进(Diemer, 1997; Cleveland \& Town- send, 2006; Ammann et al., 2007; 涂利华等, 2010; 张宇和红梅, 2014)或减缓土壤呼吸速率(贾淑霞等, 2007; 彭勇等, 2015), 但也有研究发现氮沉降对土 壤呼吸无显著影响(Allison et al., 2008)。

灌从具有种类繁多、生命力强、萌生力强、适 生范围宽等特点, 不仅在群落演替过程中扮演着极 其重要的角色, 而且在区域生态环境保护和替代能 源方面也起着非常重要的作用(刘存琦, 1994; 胡会 峰等, 2006)。然而人类的乱砍滥伐引起植被逆行演 替, 大面积森林退化形成灌从, 这种现象在华北地 区尤为显著。灌从通常为氮限制生态系统(Gorissen et al., 2004; Wessel et al., 2004)。在大气氮沉降显著 增加的情况下, 灌丛碳循环一旦发生变化, 就会对 全球气候变化产生深刻的影响。目前, 有关大气氮 沉降对灌从影响的研究十分缺乏, 氮沉降对灌从的 影响机制尚不清楚。荆条(Vitex negundo var. heterophylla)和绣线菊(Spiraea salicifolia)灌从为暖温带 分布面积广、类型典型的灌从类型(谢华辉等, 2006; 张金瑞等, 2013)。本研究选择北京东灵山地区常见 的荆条和绣线菊灌从为对象, 通过氮添加实验, 研 究东灵山灌从土壤呼吸的变化特征, 以探讨土壤呼 吸各组分对短期氮添加的初期响应特征, 并探讨其 响应机制, 以期为预测该地区在大气氮沉降持续增 加的情况下灌从土壤的碳动态提供参考。

\section{1 材料和方法}

\section{1 试验地概况}

东灵山 $\left(40.00^{\circ}-40.03^{\circ} \mathrm{N}, 115.43^{\circ}-115.50^{\circ} \mathrm{E}\right)$ 为 小五台山余脉，位于北京市西郊的门头沟区，最高 峰海拔2 303 m。该区域属于暖温带半湿润大陆季风 气候, 寒冷期长, 积温低, 年平均气温 $5-10{ }^{\circ} \mathrm{C}$, 无 霜期约195天, 年降水量500-650 mm, 多集中于夏 季(6-8月), 春旱严重(冯云等, 2007)。地带性土壤类 型为肥沃褐色土和棕色森林土。地带性植被类型以 暖温带落叶阔叶林为主。但灌从是低海拔地区最主 要的植被类型, 主要分布于海拔 $1100 \mathrm{~m}$ 以下, 其中 
荆条和绣线菊是当地的两种典型灌从, 是森林植被 遭受严重破坏后形成的次生群落(胡淑萍等, 2010)。 研究区域背景氮沉降水平仅为 $14.5 \mathrm{~kg} \mathrm{~N} \cdot \mathrm{hm}^{-2} \cdot \mathrm{a}^{-1}$, 基本没有受到污染(张建华, 2015)。荆条和绣线菊灌 从的样地情况见表 1 。

\section{2 试验设计}

2012年5月, 在荆条和绣线菊灌丛内分别选择 具有代表性、立地条件基本一致的地段, 按照随机 区组试验设计方法设置实验。在每种灌丛类型内, 分别设置 12 个 $5 \mathrm{~m} \times 5 \mathrm{~m}$ 的实验样方, 相邻样方之间 设置5-10 m的缓冲带(图1A)。在每个 $5 \mathrm{~m} \times 5 \mathrm{~m}$ 样方 中随机放置 3 个直径 $20 \mathrm{~cm}$ 的Collar环, 用于测量土 壤总呼吸 $\left(R_{\mathrm{s}}\right)$ 。通过壕沟法切断根系区分自养呼吸 和异养呼吸: 在每个样方四周挖掘 $1 \mathrm{~m}$ 深(植物根系 分布层以下)的壕沟后，用厚塑料布贴在壕沟周围 后将土回填, 隔离小区周围的根系, 以阻止根系向 小区内生长(杜恩在, 2013)。再除去样方内所有活体 植物, 设置 3 个Collar环, 用于土壤微生物呼吸的测 定(图1B), 土壤总呼吸与土壤微生物呼吸之差为根
系呼吸。

施肥梯度主要参照中国森林养分添加实验网络 (NEECF) (Du et al., 2013)。添加氮素为尿素, 共设置 4 个水平, 分别为对照 $\left(\mathrm{N}_{0}\right)$ 、低氮 $\left(\mathrm{N}_{1}\right.$, 相当于 $20 \mathrm{~kg}$ $\left.\mathrm{N} \cdot \mathrm{hm}^{-2} \cdot \mathrm{a}^{-1}\right)$ 、中氮 $\left(\mathrm{N}_{2}\right.$, 相当于 $50 \mathrm{~kg} \mathrm{~N} \cdot \mathrm{hm}^{-2} \cdot \mathrm{a}^{-1}$ ) 和高 氮 $\left(\mathrm{N}_{3}\right.$, 相当于 $\left.100 \mathrm{~kg} \mathrm{~N} \cdot \mathrm{hm}^{-2} \cdot \mathrm{a}^{-1}\right)$ 。从2012年5月开 始，一直到2013年9月在生长季(5-9月)每月月初进 行施肥。施肥主要通过肩背式喷雾器方式进行: 施 肥前将每个样地所需尿素溶于 2 L清水中(Du et al., 2013), 在样地灌从下人工均匀地进行喷酒; 每次施 肥时，对照样地 $\left(\mathrm{N}_{0}\right)$ 喷酒等量的清水。

\section{3 土壤呼吸测定}

使用LI-8100A土壤碳通量自动测量系统 (LI-COR, Lincoln, USA)观测土壤呼吸。于2012年7 月开始，每月上旬选取天气晴朗(不包括降雨后)的 9:00-11:00或13:00-16:00测定1次土壤呼吸，同时用 LI-8100自带的土壤温度和湿度传感器分别测定5 $\mathrm{cm}$ 深度的土壤体积含水量和土壤温度。每次测量时 按照不同顺序从而避免系统误差(杜恩在, 2013)。从

表1 试验样地地形、土壤和植被特征(平均值土标准误差, $n=3$ )

Table 1 Topography, soil and vegetation characteristics of the experimental sites (mean $\pm S E, n=3$ )

\begin{tabular}{|c|c|c|}
\hline 项目 & 荆条灌丛 & 绣线菊灌丛 \\
\hline Item & Vitex negundo var. heterophylla shrubland & Spiraea salicifolia shrubland \\
\hline \multicolumn{3}{|l|}{ 地形和气候 Topography and climate } \\
\hline 海拔 Elevation (m) & 791 & 1170 \\
\hline 坡向 Aspect & 南 South & 南 South \\
\hline 坡度 Slope $\left(^{\circ}\right)$ & 28 & 25 \\
\hline 年平均气温 Mean annual temperature $\left({ }^{\circ} \mathrm{C}\right)$ & 12.3 & 9.2 \\
\hline \multicolumn{3}{|l|}{ 表层土壤特征 Top soil property } \\
\hline 土壤pH值 Soil pH value & 8.7 & 8.9 \\
\hline 总碳 Total carbon $\left(\mathrm{mg} \cdot \mathrm{g}^{-1}\right)$ & $28.88 \pm 2.10$ & $39.50 \pm 5.03$ \\
\hline 总氮 Total nitrogen $\left(\mathrm{mg} \cdot \mathrm{g}^{-1}\right)$ & $2.72 \pm 0.32$ & $2.29 \pm 0.36$ \\
\hline 总磷 Total phosphorous (mg· $\mathrm{g}^{-1}$ ) & $0.47 \pm 0.04$ & $0.48 \pm 0.03$ \\
\hline 无机氮 Inorganic nitrogen (mg· $\mathrm{kg}^{-1}$ ) & $6.01 \pm 2.38$ & $2.51 \pm 2.88$ \\
\hline 速效磷 Available phosphorous (mg. $\mathrm{kg}^{-1}$ ) & $1.03 \pm 0.09$ & $1.38 \pm 0.77$ \\
\hline \multicolumn{3}{|l|}{ 群落特征 Community characteristics } \\
\hline 灌木高度 Shrub height (cm) & $78.1 \pm 12.37$ & $79.8 \pm 7.43$ \\
\hline 平均基径 Average base diameter (cm) & $0.77 \pm 1.77$ & $0.56 \pm 0.04$ \\
\hline 灌木密度 Shrub density (stems·hm ${ }^{-2}$ ) & $1.6 \times 10^{5}$ & $3.6 \times 10^{5}$ \\
\hline 灌木层优势种 Dominant species of shrub layer & $\begin{array}{l}\text { 荆条 Vitex negundo var. heterophylla, } \\
\text { 河蒴荛花 Wikstroemia chamaedaphne }\end{array}$ & 绣线菊 Spiraea salicifolia \\
\hline 草本层优势种 Dominant species of herb layer & $\begin{array}{l}\text { 细叶臺草 Carex duriuscula subsp. } \\
\text { stenophylloides }\end{array}$ & $\begin{array}{l}\text { 细叶薹草 Carex duriuscula subsp. } \\
\text { stenophylloides }\end{array}$ \\
\hline 干扰程度 Levels of disturbance & 轻度干扰 Light disturbance & 轻度干扰 Light disturbance \\
\hline
\end{tabular}




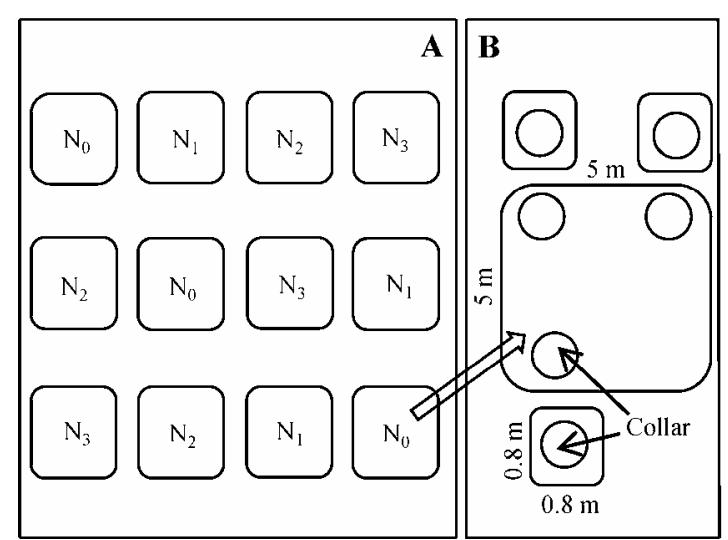

图1 北京东灵山地区灌从氮添加试验设计。A, 样方示意 图。B, Collar环示意图。 $\mathrm{N}_{0} 、 \mathrm{~N}_{1} 、 \mathrm{~N}_{2} 、 \mathrm{~N}_{3}$ 分别表示对照 $(0 \mathrm{~kg}$ $\left.\mathrm{N} \cdot \mathrm{hm}^{-2} \cdot \mathrm{a}^{-1}\right)$ 、低氮 $\left(20 \mathrm{~kg} \mathrm{~N} \cdot \mathrm{hm}^{-2} \cdot \mathrm{a}^{-1}\right)$ 、中氮 $\left(50 \mathrm{~kg} \mathrm{~N} \cdot \mathrm{hm}^{-2} \cdot \mathrm{a}^{-1}\right)$ 和高氮 $\left(100 \mathrm{~kg} \mathrm{~N} \cdot \mathrm{hm}^{-2} \cdot \mathrm{a}^{-1}\right)$ 处理。

Fig. 1 Experimental design in shrublands on Mt. Dongling, Beijing, northern China. A, Schematic diagram of Quadrats. B, Schematic diagram of Collar rings. $\mathrm{N}_{0}, \mathrm{~N}_{1}, \mathrm{~N}_{2}$ and $\mathrm{N}_{3}$ denote control $\left(0 \mathrm{~kg} \mathrm{~N} \cdot \mathrm{hm}^{-2} \cdot \mathrm{a}^{-1}\right)$, low $\left(20 \mathrm{~kg} \mathrm{~N} \cdot \mathrm{hm}^{-2} \cdot \mathrm{a}^{-1}\right)$, medium (50 $\left.\mathrm{kg} \mathrm{N} \cdot \mathrm{hm}^{-2} \cdot \mathrm{a}^{-1}\right)$, and high $\left(100 \mathrm{~kg} \mathrm{~N} \cdot \mathrm{hm}^{-2} \cdot \mathrm{a}^{-1}\right)$ nitrogen addition, respectively.

2012年4月开始, 在每种灌丛类型地下5 cm各放置 一个Stow Away Tidbit Temp Logger土壤温度记录仪 (Onset Computer, Bourne, USA), 连续记录土壤温 度, 采样间隔为 $1 \mathrm{~h}$ 。

\section{4 数据分析}

土壤呼吸速率 $(R)\left(\mu \mathrm{mol} \mathrm{CO}_{2} \cdot \mathrm{m}^{-2} \cdot \mathrm{s}^{-1}\right)$ 和 $5 \mathrm{~cm}$ 土 壤温度 $(T)$ 以及 $0-5 \mathrm{~cm}$ 土壤体积含水率 $(V W C)$ 的拟合 关系如下:

$$
\begin{aligned}
& R=a \mathrm{e}^{K T} \\
& R=a V W C+b
\end{aligned}
$$

并基于上述公式(1)推导出全年土壤呼吸温度 敏感性系数 $\left(Q_{10}\right)$ :

$$
Q_{10}=\mathrm{e}^{10 K}
$$

$a 、 b 、 K$ 为拟合参数。

根据土壤呼吸速率与土壤温度的指数关系(公 式(1)), 通过样地内放置的温度自动记录仪记录的 土壤温度, 分别计算各样地年平均土壤呼吸速率和 单位面积土壤 $\mathrm{CO}_{2}$ 通量为:

$$
\begin{aligned}
& R_{\mathrm{d}}=\sum_{1}^{24} R_{\mathrm{i}} \times 3600 \times 10^{4} \times 12 \times 10^{-6} \times 10^{-6} \\
& R_{\mathrm{y}}=\sum_{1}^{365} R_{\mathrm{d}}
\end{aligned}
$$

$R_{\mathrm{i}} 、 R_{\mathrm{d}}$ 和 $R_{\mathrm{y}}$ 分别为土壤呼吸速率 $\left(\mu \mathrm{mol} \mathrm{CO} \mathrm{CO}^{-2} \cdot \mathrm{m}^{-1}\right)$ 、 土壤呼吸日通量 $\left(\mathrm{t} \mathrm{C} \cdot \mathrm{m}^{-2} \cdot \mathrm{d}^{-1}\right)$ 和年通量 $\left(\mathrm{t} \mathrm{C} \cdot \mathrm{hm}^{-2} \cdot \mathrm{a}^{-1}\right)$ 。
采用重复单因素方差分析比较不同氮添加水平 对土壤呼吸的影响。

方差分析在软件SPSS 17.0中完成, 图表绘制均 在Word 2003、Excel 2003和SigmaPlot 12.0内完成。

\section{2 结果和分析}

\section{1 氮添加对土壤总呼吸季节变化的影响}

荆条和绣线菊灌从的土壤总呼吸存在明显的季 节格局(表1, $p<0.001$ ), 总体呈单峰型。2012年的峰 值均出现在 8 月, 2013年的峰值出现在7月(图2A, 2D)。重复测量方差分析结果显示, 在生长季, 施肥 对荆条灌从和绣线菊灌从土壤总呼吸均无显著影响 $(p>0.05)$ (表2)。

荆条和绣线菊灌从土壤总呼吸与土壤温度呈显 著的指数关系(图3)。两灌从对氮添加的响应不同, 具体表现在, 低高氮 $\left(\mathrm{N}_{1}, \mathrm{~N}_{3}\right)$ 处理和中氮 $\left(\mathrm{N}_{2}\right)$ 处理在 一定程度上分别提高和降低了荆条灌从土壤呼吸 $Q_{10}$, 而绣线菊灌从在相应氮处理的土壤呼吸 $Q_{10}$ 的 变化却表现出相反趋势。 $\mathrm{N}_{0} 、 \mathrm{~N}_{1} 、 \mathrm{~N}_{2} 、 \mathrm{~N}_{3}$ 处理下荆 条和绣线菊灌从土壤总呼吸的 $Q_{10}$ 分别为 $1.52 、 1.58$ 、 $1.44 、 1.55$ 和 $1.54 、 1.43 、 1.98 、 1.44$ (表3)。施肥没 有显著改变 $Q_{10}$ 。各处理水平的土壤总呼吸与土壤水 分均显著正相关(图4)。

\section{2 氮添加对土壤异养呼吸的影响}

荆条和绣线菊灌从的异养呼吸存在明显的季节 格局, 总体呈单峰型。荆条灌从的土壤异养呼吸在 2012和2013年的峰值分别出现在 8月和7月(图2B, $2 \mathrm{E})$, 而绣线菊灌从的峰值均出现在7月。各处理对 荆条灌从的异养呼吸无显著影响(图2B), 在生长季, $\mathrm{N}_{2}$ 和 $\mathrm{N}_{3}$ 处理显著地促进了绣线菊灌从的异养呼吸, 而 $\mathrm{N}_{1}$ 处理效果不显著(图2E; 表2)。

各氮处理水平下, 荆条和绣线菊灌从土壤异养 呼吸与土壤温度呈显著的指数关系(图5; 表3)。氮添 加对两灌从土壤异养呼吸温度敏感性的影响不同, $\mathrm{N}_{1}$ 和 $\mathrm{N}_{3}$ 处理提高了荆条灌从土壤异养呼吸温度敏 感性，而 $\mathrm{N}_{2}$ 处理降低了其温度敏感性; $\mathrm{N}_{0} 、 \mathrm{~N}_{1} 、 \mathrm{~N}_{2}$ 、 $\mathrm{N}_{3}$ 处理下的 $Q_{10}$ 分别为1.42、2.11、1.38、1.78 (图5; 表 3)。 $N_{1}$ 和 $N_{3}$ 处理降低了绣线菊灌从土壤异养呼吸温 度敏感性，而 $\mathrm{N}_{2}$ 处理提高了其温度敏感性; $\mathrm{N}_{0} 、 \mathrm{~N}_{1}$ 、 $\mathrm{N}_{2} 、 \mathrm{~N}_{3}$ 处理下的 $Q_{10}$ 分别为 $1.63 、 1.49 、 1.88 、 1.59$ 。 各氮处理水平下, 荆条和绣线菊灌从的土壤异养呼 吸与土壤水分均显著正相关(图6)。 

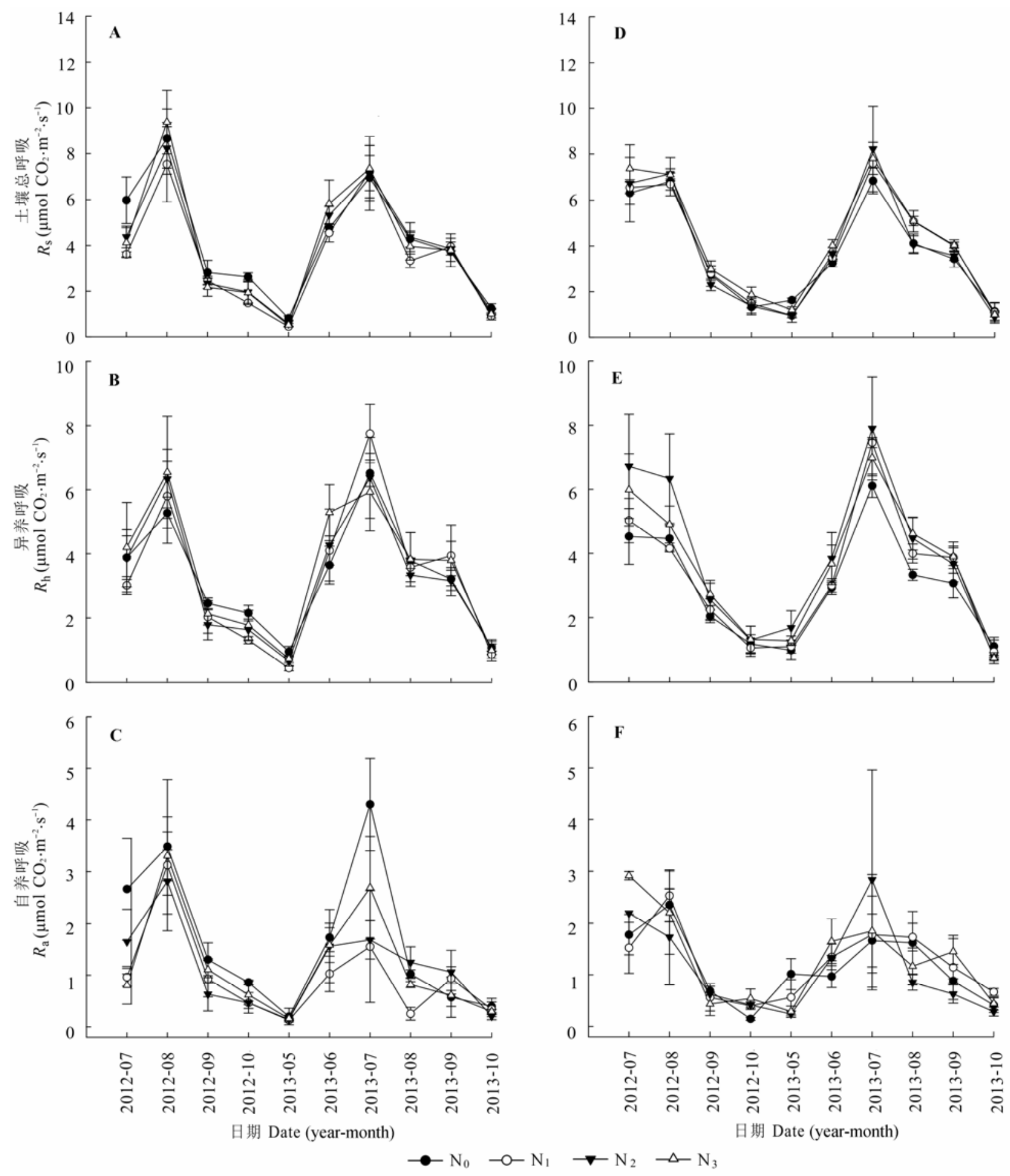

图2 不同氮添加对荆条(左)和绣线菊(右)灌从土壤总呼吸 $\left(R_{\mathrm{s}}\right)$ 、自养呼吸 $\left(R_{\mathrm{a}}\right)$ 及异养呼吸 $\left(R_{\mathrm{h}}\right)$ 的影响(平均值土标准误差)。 $\mathrm{N}_{0}$ 、 $\mathrm{N}_{1} 、 \mathrm{~N}_{2} 、 \mathrm{~N}_{3}$ 分别表示对照 $\left(0 \mathrm{~kg} \mathrm{~N} \cdot \mathrm{hm}^{-2} \cdot \mathrm{a}^{-1}\right)$ 、低氮 $\left(20 \mathrm{~kg} \mathrm{~N} \cdot \mathrm{hm}^{-2} \cdot \mathrm{a}^{-1}\right)$ 、中氮 $\left(50 \mathrm{~kg} \mathrm{~N} \cdot \mathrm{hm}^{-2} \cdot \mathrm{a}^{-1}\right)$ 和高氮 $\left(100 \mathrm{~kg} \mathrm{~N} \cdot \mathrm{hm} \mathrm{m}^{-2} \cdot \mathrm{a}^{-1}\right)$ 处理。 Fig. 2 Influence of different nitrogen addition on the soil total respiration $\left(R_{\mathrm{s}}\right)$, autotrophic respiration $\left(R_{\mathrm{a}}\right)$ and heterotrophic respiration $\left(R_{\mathrm{h}}\right)$ of Vitex negundo var. heterophylla (left) and Spiraea salicifolia (right) shrublands (mean $\pm S E$ ). $\mathrm{N}_{0}, \mathrm{~N}_{1}, \mathrm{~N}_{2}$ and $\mathrm{N}_{3}$ denote control $\left(0 \mathrm{~kg} \mathrm{~N} \cdot \mathrm{hm}^{-2} \cdot \mathrm{a}^{-1}\right)$, low $\left(20 \mathrm{~kg} \mathrm{~N} \cdot \mathrm{hm}^{-2} \cdot \mathrm{a}^{-1}\right)$, medium $\left(50 \mathrm{~kg} \mathrm{~N} \cdot \mathrm{hm}^{-2} \cdot \mathrm{a}^{-1}\right)$, and high $\left(100 \mathrm{~kg} \mathrm{~N} \cdot \mathrm{hm}{ }^{-2} \cdot \mathrm{a}^{-1}\right)$ nitrogen addition, respectively.

\section{3 氮添加对土壤自养呼吸的影响}

荆条和绣线菊灌从的土壤自养呼吸均存在明显 的季节格局, 且变化趋势相似, 总体呈单峰型。2012
年的峰值均出现在8月, 2013年的峰值出现在7月(图 2C、2F)。施氮降低了荆条灌丛土壤的自养呼吸, 其 中 $\mathrm{N}_{1}$ 处理对荆条灌丛土壤自养呼吸表现为显著的 
表2 时间和氮添加处理对荆条和绣线菊灌从土壤总呼吸 $\left(R_{\mathrm{s}}\right)$ 、异养呼吸 $\left(R_{\mathrm{h}}\right)$ 及自养呼吸 $\left(R_{\mathrm{a}}\right)$ 影响的双因子方差分析

Table 2 Two-way ANOVA test results for the effects of time and nitrogen addition treatments on total soil respiration $\left(R_{\mathrm{s}}\right)$, heterotrophic respiration $\left(R_{\mathrm{h}}\right)$ and autotrophic respiration rate $\left(R_{\mathrm{a}}\right)$ in Vitex negundo var. heterophylla and Spiraea salicifolia shrublands

\begin{tabular}{|c|c|c|c|c|c|c|c|}
\hline & \multirow{2}{*}{$\begin{array}{l}\text { 自由度 } \\
\text { Degree of } \\
\text { freedom }\end{array}$} & \multicolumn{2}{|c|}{$R_{\mathrm{S}}$} & \multicolumn{2}{|c|}{$R_{\mathrm{h}}$} & \multicolumn{2}{|c|}{$R_{\mathrm{a}}$} \\
\hline & & $F$ & $p$ & $F$ & $p$ & $F$ & $p$ \\
\hline \multicolumn{8}{|c|}{ 荆条灌丛 Vitex negundo var. heterophylla shrubland } \\
\hline 处理 Treatment & 3 & 1.51 & 0.218 & 0.33 & 0.807 & 3.33 & 0.024 \\
\hline 时间 Time ${ }^{1)}$ & 9 & 52.49 & $<0.001$ & 33.18 & $<0.001$ & 14.60 & $<0.001$ \\
\hline 处理 $\times$ 时间 Treatment $\times$ Time & 27 & 0.42 & 0.994 & 0.45 & 0.989 & 0.88 & 0.631 \\
\hline \multicolumn{8}{|c|}{ 绣线菊灌从 Spiraea salicifolia shrubland } \\
\hline 处理 Treatment & 3 & 1.49 & 0.224 & 4.43 & 0.006 & 0.42 & 0.740 \\
\hline 时间 Time & 9 & 88.65 & $<0.001$ & 42.90 & $<0.001$ & 7.25 & $<0.001$ \\
\hline 处理 $\times$ 时间 Treatment $\times$ Time & 27 & 0.39 & 0.996 & 0.49 & 0.980 & 0.32 & 0.999 \\
\hline
\end{tabular}

1) 土壤呼吸和异养呼吸的测量时间。

1) measuring time of soil and heterotrophic respiration.
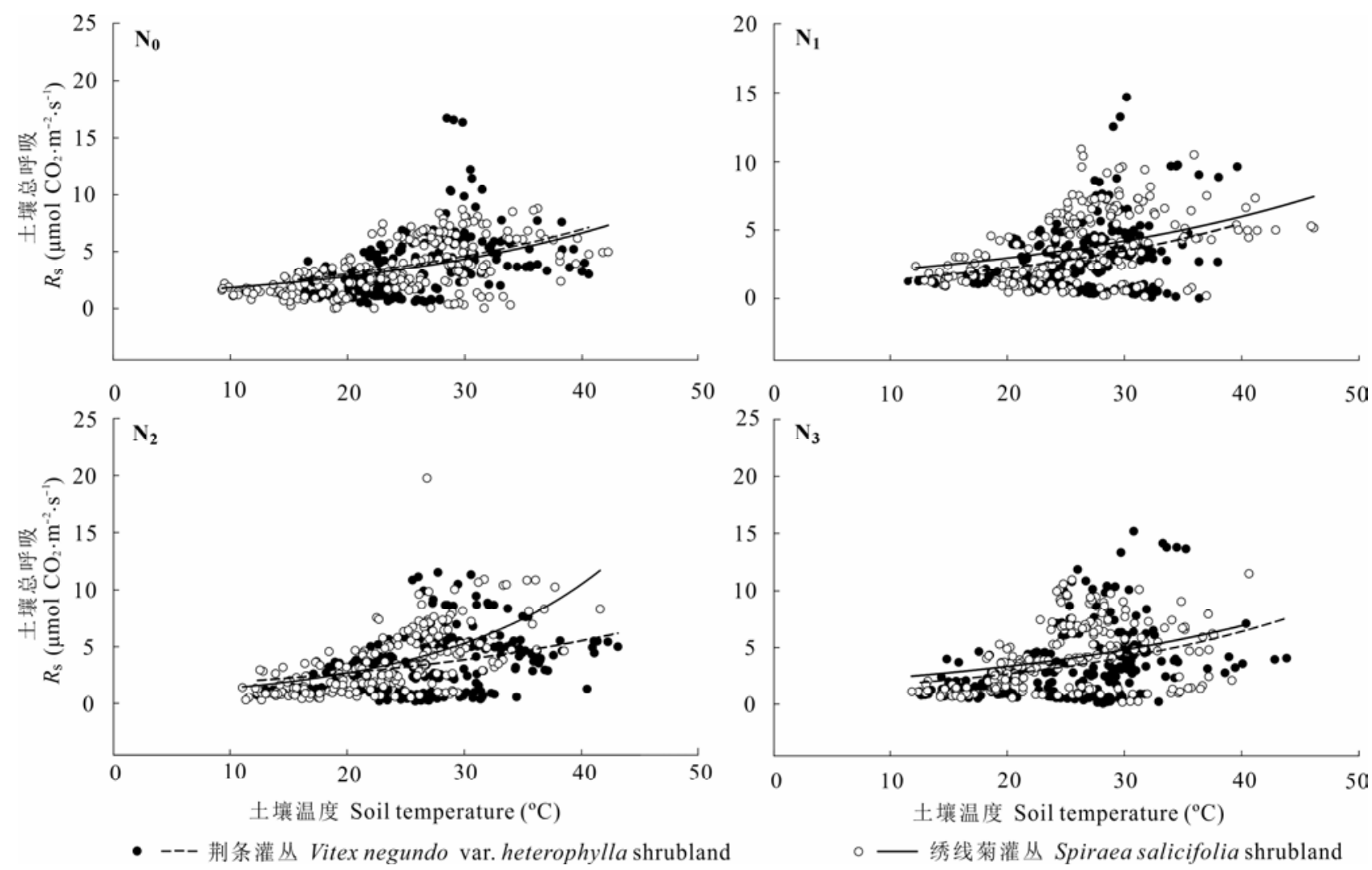

图3 不同氮添加处理的荆条和绣线菊灌从土壤总呼吸 $\left(R_{\mathrm{s}}\right)$ 与土壤温度的关系。 $\mathbf{N}_{0} 、 \mathbf{N}_{1} 、 \mathbf{N}_{2} 、 \mathbf{N}_{3}$ 分别表示对照 $\left(0 \mathrm{~kg} \mathrm{~N} \cdot \mathrm{hm} \mathrm{m}^{-2} \cdot \mathrm{a}^{-1}\right)$ 、 低氮 $\left(20 \mathrm{~kg} \mathrm{~N} \cdot \mathrm{hm}^{-2} \cdot \mathrm{a}^{-1}\right)$ 、中氮 $\left(50 \mathrm{~kg} \mathrm{~N} \cdot \mathrm{hm}^{-2} \cdot \mathrm{a}^{-1}\right)$ 和高氮 $\left(100 \mathrm{~kg} \mathrm{~N} \cdot \mathrm{hm}^{-2} \cdot \mathrm{a}^{-1}\right)$ 处理。

Fig. 3 Relationship between total soil respiration $\left(R_{\mathrm{s}}\right)$ and soil temperature in Vitex negundo var. heterophylla and Spiraea salicifolia shrublands under different nitrogen addition treatments. $\mathbf{N}_{\mathbf{0}}, \mathbf{N}_{\mathbf{1}}, \mathbf{N}_{2}$ and $\mathbf{N}_{3}$ denote control $\left(0 \mathrm{~kg} \mathrm{~N} \cdot \mathrm{hm}^{-2} \cdot \mathrm{a}^{-1}\right), \operatorname{low}\left(20 \mathrm{~kg} \mathrm{~N} \cdot \mathrm{hm}{ }^{-2} \cdot \mathrm{a}^{-1}\right)$, medium $\left(50 \mathrm{~kg} \mathrm{~N} \cdot \mathrm{hm}^{-2} \cdot \mathrm{a}^{-1}\right)$, and high $\left(100 \mathrm{~kg} \mathrm{~N} \cdot \mathrm{hm}^{-2} \cdot \mathrm{a}^{-1}\right)$ nitrogen addition, respectively.

抑制效应。在生长季，氮添加对绣线菊灌从的自养 呼吸无明显的影响(图2C、2F)。

\section{4 氮添加对土壤呼吸年通量的影响}

在荆条灌从样地, $\mathrm{N}_{0} 、 \mathrm{~N}_{1} 、 \mathrm{~N}_{2}$ 和 $\mathrm{N}_{3}$ 处理的土壤 总呼吸年碳排放量分别为: 5.91、7.93、5.30和5.09 t $\mathrm{C} \cdot \mathrm{hm}^{-2} \cdot \mathrm{a}^{-1}$, 低氮处理的排放比对照高出 $34.1 \%$, 中
氮和高氮处理的排放量几乎相同，排放量均低于对 照, 约降低了 $10.4 \%$; 氮添加降低了异养呼吸, 各氮 处理的异养呼吸年排放量分别为: 5.76、3.56、5.04 和 $4.25 \mathrm{t} \mathrm{C} \cdot \mathrm{hm}^{-2} \cdot \mathrm{a}^{-1}$ (图7A、7B)。方差分析结果表明, 氮添加对荆条灌从土壤呼吸、异养呼吸年通量均无 显著影响。 
表3 土壤呼吸 $\left(R, \mu \mathrm{mol} \mathrm{CO} \mathrm{CO}^{-2} \cdot \mathrm{m}^{-1}\right)$ 和组分与 $5 \mathrm{~cm}$ 土壤温度 $\left.\left(T,{ }^{\circ} \mathrm{C}\right)\right)$ 的指数关系模型 $\left(R=a e^{K t}\right)$

Table 3 Models $\left(R=a e^{K t}\right)$ for the relationship between total and component of soil respiration $\left(R, \mu \mathrm{mol} \mathrm{CO}_{2} \cdot \mathrm{m}^{-2} \cdot \mathrm{s}^{-1}\right)$ and soil temperature $5 \mathrm{~cm}$ under the surface

\begin{tabular}{|c|c|c|c|c|c|c|c|}
\hline \multirow{2}{*}{$\begin{array}{l}\text { 灌从类型 } \\
\text { Shrubland type }\end{array}$} & \multirow{2}{*}{$\begin{array}{l}\text { 呼吸组分 } \\
\text { Respiration component }\end{array}$} & \multirow{2}{*}{$\begin{array}{l}\text { 处理 } \\
\text { Treatment }\end{array}$} & \multirow[t]{2}{*}{$n$} & \multirow[t]{2}{*}{$R^{2}$} & \multicolumn{3}{|c|}{ 参数 Parameter } \\
\hline & & & & & $\bar{a}$ & K & $Q_{10}$ \\
\hline \multirow{8}{*}{ 荆条 Vitex negundo var. heterophylla } & 土壤总呼吸 & $\mathrm{N}_{0}$ & 217 & 0.13 & 1.322 & 0.042 & 1.52 \\
\hline & \multirow[t]{3}{*}{ Total soil respiration } & $\mathrm{N}_{1}$ & 227 & 0.08 & 0.878 & 0.046 & 1.58 \\
\hline & & $\mathrm{N}_{2}$ & 231 & 0.13 & 1.296 & 0.036 & 1.44 \\
\hline & & $\mathrm{N}_{3}$ & 234 & 0.12 & 1.109 & 0.044 & 1.55 \\
\hline & 异养呼吸 & $\mathrm{N}_{0}$ & 220 & 0.20 & 1.183 & 0.035 & 1.42 \\
\hline & \multirow[t]{3}{*}{ Heterotrophic respiration } & $\mathrm{N}_{1}$ & 221 & 0.26 & 0.327 & 0.075 & 2.11 \\
\hline & & $\mathrm{N}_{2}$ & 224 & 0.14 & 1.137 & 0.032 & 1.38 \\
\hline & & $\mathrm{N}_{3}$ & 242 & 0.18 & 0.594 & 0.058 & 1.78 \\
\hline \multirow[t]{8}{*}{ 绣线菊 Spiraea salicifolia } & 土壤总呼吸 & $\mathrm{N}_{0}$ & 228 & 0.28 & 1.192 & 0.043 & 1.54 \\
\hline & \multirow[t]{3}{*}{ Total soil respiration } & $\mathrm{N}_{1}$ & 218 & 0.16 & 1.445 & 0.036 & 1.43 \\
\hline & & $\mathrm{N}_{2}$ & 232 & 0.39 & 0.678 & 0.069 & 1.98 \\
\hline & & $\mathrm{N}_{3}$ & 216 & 0.15 & 1.609 & 0.037 & 1.44 \\
\hline & 异养呼吸 & $\mathrm{N}_{0}$ & 224 & 0.33 & 0.753 & 0.049 & 1.63 \\
\hline & \multirow[t]{3}{*}{ Heterotrophic respiration } & $\mathrm{N}_{1}$ & 221 & 0.22 & 1.003 & 0.040 & 1.49 \\
\hline & & $\mathrm{N}_{2}$ & 234 & 0.39 & 0.666 & 0.063 & 1.88 \\
\hline & & $\mathrm{N}_{3}$ & 213 & 0.31 & 0.942 & 0.047 & 1.59 \\
\hline
\end{tabular}

$\mathrm{N}_{0} 、 \mathrm{~N}_{1} 、 \mathrm{~N}_{2} 、 \mathrm{~N}_{3}$ 分别表示对照 $\left(0 \mathrm{~kg} \mathrm{~N} \cdot \mathrm{hm}^{-2} \cdot \mathrm{a}^{-1}\right)$ 、低氮 $\left(20 \mathrm{~kg} \mathrm{~N} \cdot \mathrm{hm}^{-2} \cdot \mathrm{a}^{-1}\right)$ 、中氮 $\left(50 \mathrm{~kg} \mathrm{~N} \cdot \mathrm{hm}^{-2} \cdot \mathrm{a}^{-1}\right)$ 和高氮 $\left(100 \mathrm{~kg} \mathrm{~N} \cdot \mathrm{hm}^{-2} \cdot \mathrm{a}^{-1}\right)$ 处理。 $Q_{10}$, 温度敏感性系数。 $\mathrm{N}_{0}, \mathrm{~N}_{1}, \mathrm{~N}_{2}$ and $\mathrm{N}_{3}$ denote control $\left(0 \mathrm{~kg} \mathrm{~N} \cdot \mathrm{hm}^{-2} \cdot \mathrm{a}^{-1}\right)$, low $\left(20 \mathrm{~kg} \mathrm{~N} \cdot \mathrm{hm}^{-2} \cdot \mathrm{a}^{-1}\right)$, medium $\left(50 \mathrm{~kg} \mathrm{~N} \cdot \mathrm{hm}^{-2} \cdot \mathrm{a}^{-1}\right)$, and high $\left(100 \mathrm{~kg} \mathrm{~N} \cdot \mathrm{hm}^{-2} \cdot \mathrm{a}^{-1}\right)$ nitrogen addition, respectively. $Q_{10}$, temperature sensitivity.
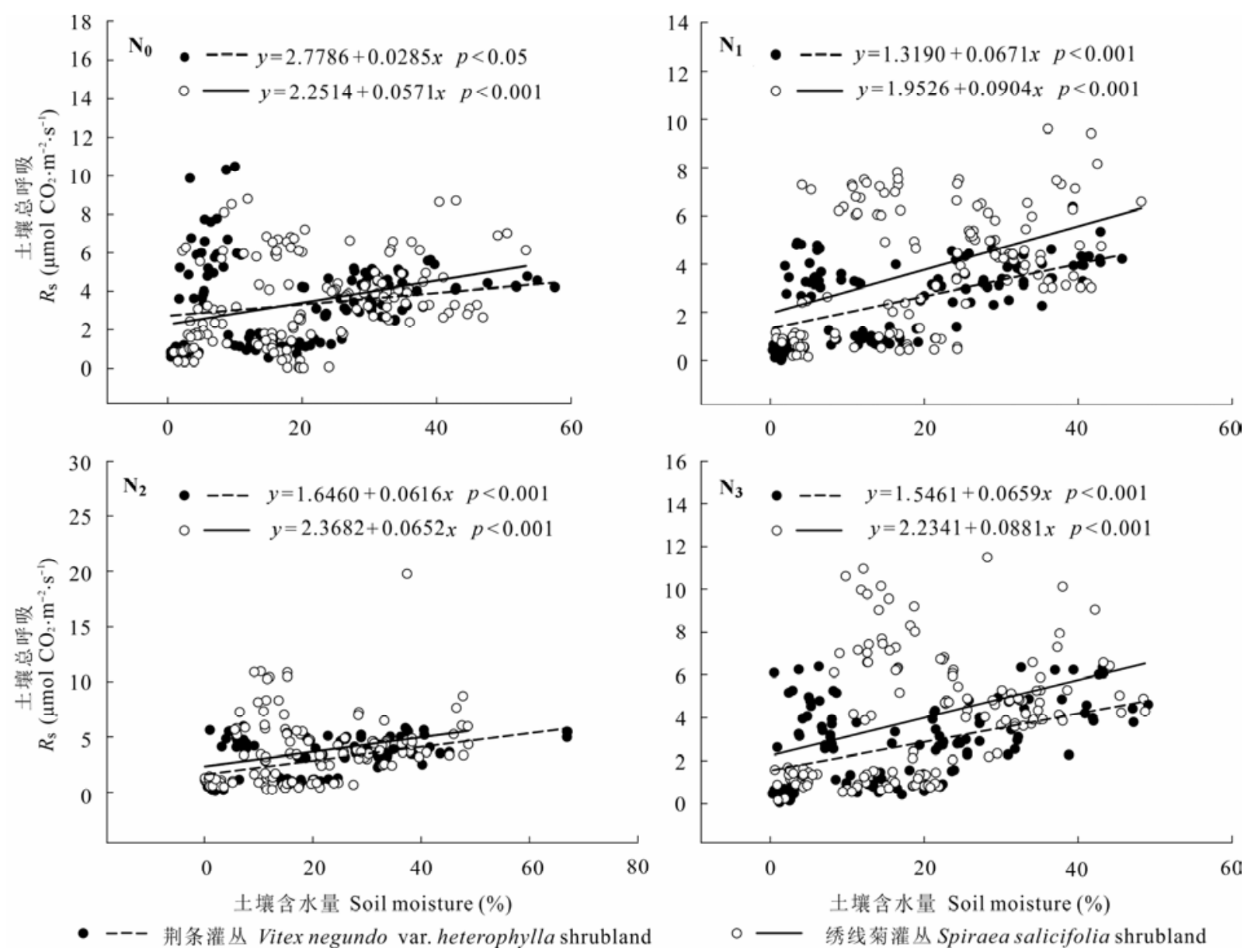

图4 不同处理的荆条和绣线菊灌丛土壤总呼吸 $\left(R_{\mathrm{s}}\right)$ 与土壤含水量的关系。 $\mathbf{N}_{\mathbf{0}} 、 \mathbf{N}_{1} 、 \mathbf{N}_{2} 、 \mathbf{N}_{3}$ 分别表示对照 $\left(0 \mathrm{~kg} \mathrm{~N} \cdot \mathrm{hm} \mathrm{m}^{-2} \cdot \mathrm{a}^{-1}\right)$ 、 低氮 $\left(20 \mathrm{~kg} \mathrm{~N} \cdot \mathrm{hm}^{-2} \cdot \mathrm{a}^{-1}\right)$ 、中氮 $\left(50 \mathrm{~kg} \mathrm{~N} \cdot \mathrm{hm}^{-2} \cdot \mathrm{a}^{-1}\right)$ 和高氮 $\left(100 \mathrm{~kg} \mathrm{~N} \cdot \mathrm{hm}^{-2} \cdot \mathrm{a}^{-1}\right)$ 处理。

Fig. 4 Relationship between total soil respiration $\left(R_{s}\right)$ and soil moisture in Vitex negundo var. heterophylla and Spiraea salicifolia shrublands under different nitrogen addition treatments. $\mathbf{N}_{\mathbf{0}}, \mathbf{N}_{\mathbf{1}}, \mathbf{N}_{2}$ and $\mathbf{N}_{3}$ denote control $\left(0 \mathrm{~kg} \mathrm{~N} \cdot \mathrm{hm}^{-2} \cdot \mathrm{a}^{-1}\right), \operatorname{low}\left(20 \mathrm{~kg} \mathrm{~N} \cdot \mathrm{hm}{ }^{-2} \cdot \mathrm{a}^{-1}\right)$, medium $\left(50 \mathrm{~kg} \mathrm{~N} \cdot \mathrm{hm}^{-2} \cdot \mathrm{a}^{-1}\right)$, and high $\left(100 \mathrm{~kg} \mathrm{~N} \cdot \mathrm{hm}^{-2} \cdot \mathrm{a}^{-1}\right)$ nitrogen addition, respectively. 

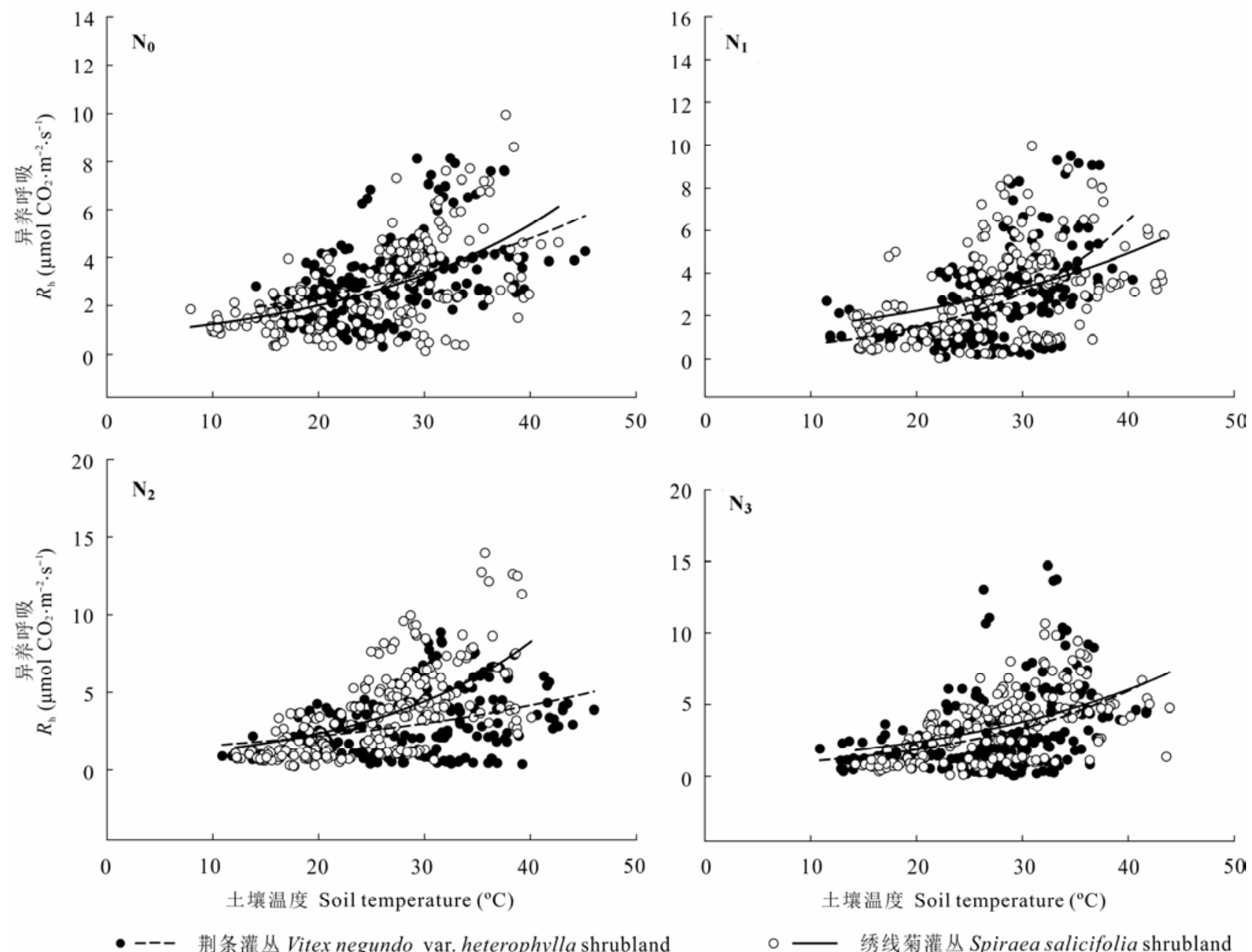

图5 不同氮添加处理的荆条和绣线菊灌从土壤异养呼吸 $\left(R_{\mathrm{h}}\right)$ 与土壤温度的关系。 $\mathbf{N}_{\mathbf{0}} 、 \mathbf{N}_{1} 、 \mathbf{N}_{2} 、 \mathbf{N}_{3}$ 分别表示对照 $(0 \mathrm{~kg}$ $\left.\mathrm{N} \cdot \mathrm{hm}^{-2} \cdot \mathrm{a}^{-1}\right)$ 、低氮 $\left(20 \mathrm{~kg} \mathrm{~N} \cdot \mathrm{hm}^{-2} \cdot \mathrm{a}^{-1}\right)$ 、中氮 $\left(50 \mathrm{~kg} \mathrm{~N} \cdot \mathrm{hm}^{-2} \cdot \mathrm{a}^{-1}\right)$ 和高氮 $\left(100 \mathrm{~kg} \mathrm{~N} \cdot \mathrm{hm}^{-2} \cdot \mathrm{a}^{-1}\right)$ 处理。

Fig. 5 Relationship between soil heterotrophic respiration $\left(R_{\mathrm{h}}\right)$ and soil temperature in Vitex negundo var. heterophylla and Spiraea salicifolia shrublands under different nitrogen addition treatments. $\mathbf{N}_{\mathbf{0}}, \mathbf{N}_{\mathbf{1}}, \mathbf{N}_{\mathbf{2}}$ and $\mathbf{N}_{\mathbf{3}}$ denote control $\left(0 \mathrm{~kg} \mathrm{~N} \cdot \mathrm{hm} \mathrm{m}^{-2} \cdot \mathrm{a}^{-1}\right)$, low $(20 \mathrm{~kg}$ $\left.\mathrm{N} \cdot \mathrm{hm}^{-2} \cdot \mathrm{a}^{-1}\right)$, medium $\left(50 \mathrm{~kg} \mathrm{~N} \cdot \mathrm{hm}^{-2} \cdot \mathrm{a}^{-1}\right)$, and high $\left(100 \mathrm{~kg} \mathrm{~N} \cdot \mathrm{hm}^{-2} \cdot \mathrm{a}^{-1}\right)$ nitrogen addition, respectively.

在绣线菊样地, 各氮添加样地的土壤总呼吸年 碳排放量分别为: 4.23、3.60、3.60和5.99 $\mathrm{t} \mathrm{C} \cdot \mathrm{hm}^{-2} \cdot \mathrm{a}^{-1}$; 高氮处理的排放比对照高出 $41.7 \%$; 中氮和低氮处 理的排放量相同，均低于对照，约降低了 $14.7 \%$; 各 氮添加样地的异养呼吸同对照组很接近(图7A、 $7 B)$ 。氮添加对绣线菊灌从土壤总呼吸、异养呼吸年 通量均无显著的影响。

从图8和表4可知, 荆条样地对照组中异养呼吸 占总呼吸比例最高 $(95.0 \%)$; 中氮处理 $\left(\mathrm{N}_{2}\right)$ 下异养呼 吸所占比例与对照组的接近; 低氮处理 $\left(\mathrm{N}_{1}\right)$ 的异养 呼吸占总呼吸的比例最低( $47.0 \%)$ 。在绣线菊样地, 中氮 $\left(\mathrm{N}_{2}\right)$ 处理样地的异养呼吸占总呼吸的比例最高 (95.0\%), 高氮 $\left(\mathrm{N}_{3}\right)$ 处理的异养呼吸贡献最低 (54.5\%)。

\section{3 讨论}

土壤呼吸在不同气候带和植被类型存在很大差 异, 并与净初级生产力密切相关(涂丽华等, 2011)。 本研究结果表明, 东灵山荆条和绣线菊灌从土壤总 呼吸的年通量分别为5.91和4.23 t C $\cdot \mathrm{hm}^{-2} \cdot \mathrm{a}^{-1}$, 均显 著低于南亚热带鼎湖山季风常绿阔叶林 $(10.80$ $\left.\mathrm{t} \mathrm{C} \cdot \mathrm{hm}^{-2} \cdot \mathrm{a}^{-1}\right)$ 、北亚热带-南暖温带过渡区锐齿栋 (Quercus aliena var. acuteserrata)老林(7.79 t C $\cdot \mathrm{hm}^{-2}$. $\left.\mathrm{a}^{-1}\right)$ 、北亚和热带北缘锐齿栋林 $\left(7.71 \mathrm{tC} \cdot \mathrm{hm}^{-2} \cdot \mathrm{a}^{-1}\right)$ (胡 正华等, 2010), 但与当地白华(Betula platyphylla)林 (5.74 t C $\cdot \mathrm{hm}^{-2} \cdot \mathrm{a}^{-1}$ )、辽东栋(Quercus mongolica)林 (4.55 t C $\cdot \mathrm{hm}^{-2} \cdot \mathrm{a}^{-1}$ )和油松(Pinus tabuliformis)林(4.14 $\mathrm{t} \mathrm{C} \cdot \mathrm{hm}^{-2} \cdot \mathrm{a}^{-1}$ ) (姚辉等, 2015)的土壤总呼吸相差不 

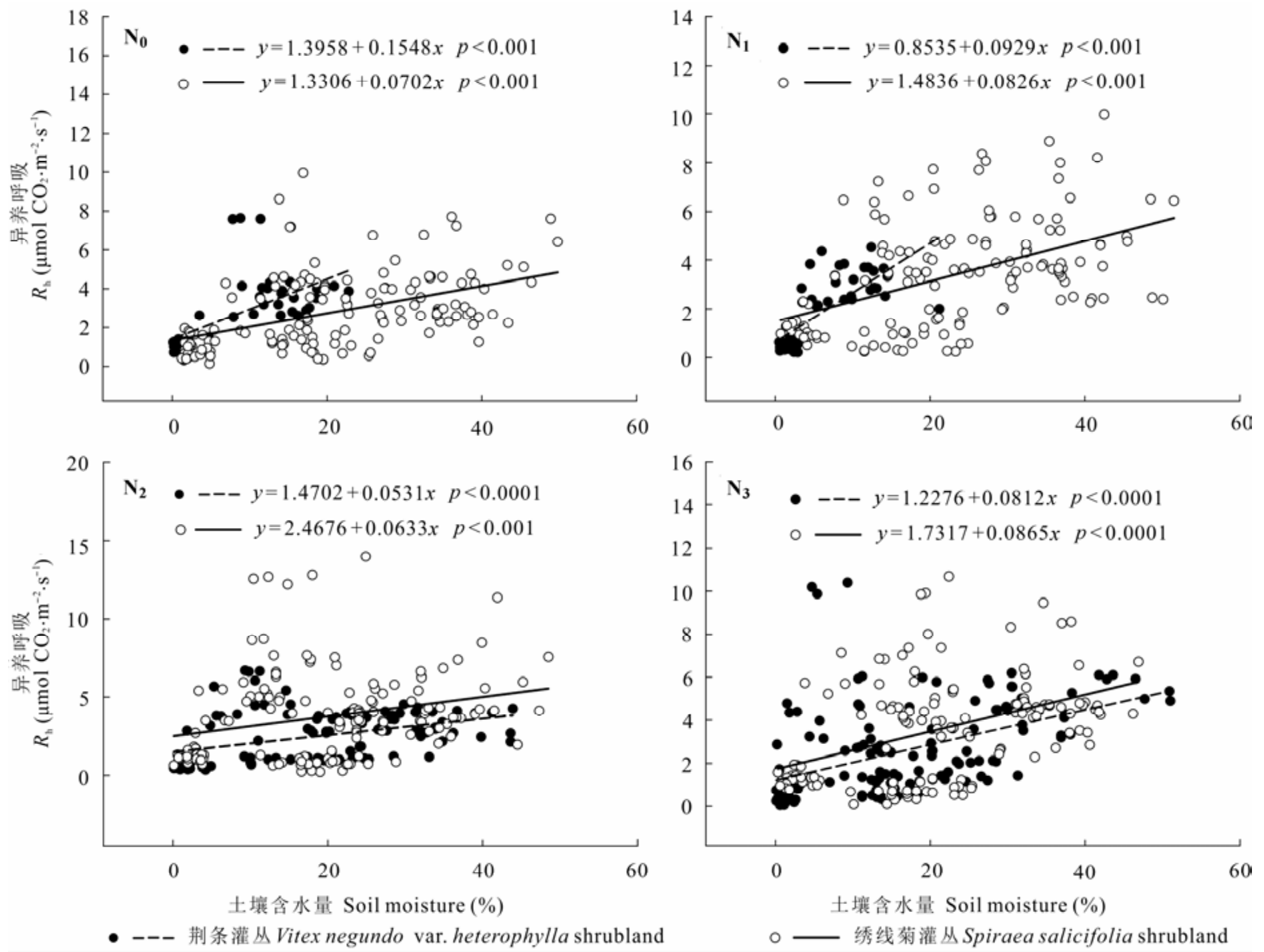

图6 不同氮处理的荆条和绣线菊灌从土壤异养呼吸 $\left(R_{\mathrm{h}}\right)$ 与土壤含水量的关系。 $\mathbf{N}_{\mathbf{0}} 、 \mathbf{N}_{1} 、 \mathbf{N}_{2} 、 \mathbf{N}_{3}$ 分别表示对照 $\left(0 \mathrm{~kg} \mathrm{~N} \cdot \mathrm{hm} \mathrm{m}^{-2} \cdot \mathrm{a}^{-1}\right)$ 、 低氮 $\left(20 \mathrm{~kg} \mathrm{~N} \cdot \mathrm{hm}^{-2} \cdot \mathrm{a}^{-1}\right)$ 、中氮 $\left(50 \mathrm{~kg} \mathrm{~N} \cdot \mathrm{hm}^{-2} \cdot \mathrm{a}^{-1}\right)$ 和高氮 $\left(100 \mathrm{~kg} \mathrm{~N} \cdot \mathrm{hm}^{-2} \cdot \mathrm{a}^{-1}\right)$ 处理。

Fig. 6 Relationship between soil heterotrophic respiration $\left(R_{\mathrm{h}}\right)$ and soil moisture in Vitex negundo var. heterophylla and Spiraea salicifolia shrublands under different nitrogen addition treatments. $\mathbf{N}_{\mathbf{0}}, \mathbf{N}_{\mathbf{1}}, \mathbf{N}_{\mathbf{2}}$ and $\mathbf{N}_{\mathbf{3}}$ denote control $\left(0 \mathrm{~kg} \mathrm{~N} \cdot \mathrm{hm} \mathrm{m}^{-2} \cdot \mathrm{a}^{-1}\right)$, low $(20 \mathrm{~kg}$ $\left.\mathrm{N} \cdot \mathrm{hm}^{-2} \cdot \mathrm{a}^{-1}\right)$, medium $\left(50 \mathrm{~kg} \mathrm{~N} \cdot \mathrm{hm}^{-2} \cdot \mathrm{a}^{-1}\right)$, and high $\left(100 \mathrm{~kg} \mathrm{~N} \cdot \mathrm{hm}^{-2} \cdot \mathrm{a}^{-1}\right)$ nitrogen addition, respectively.
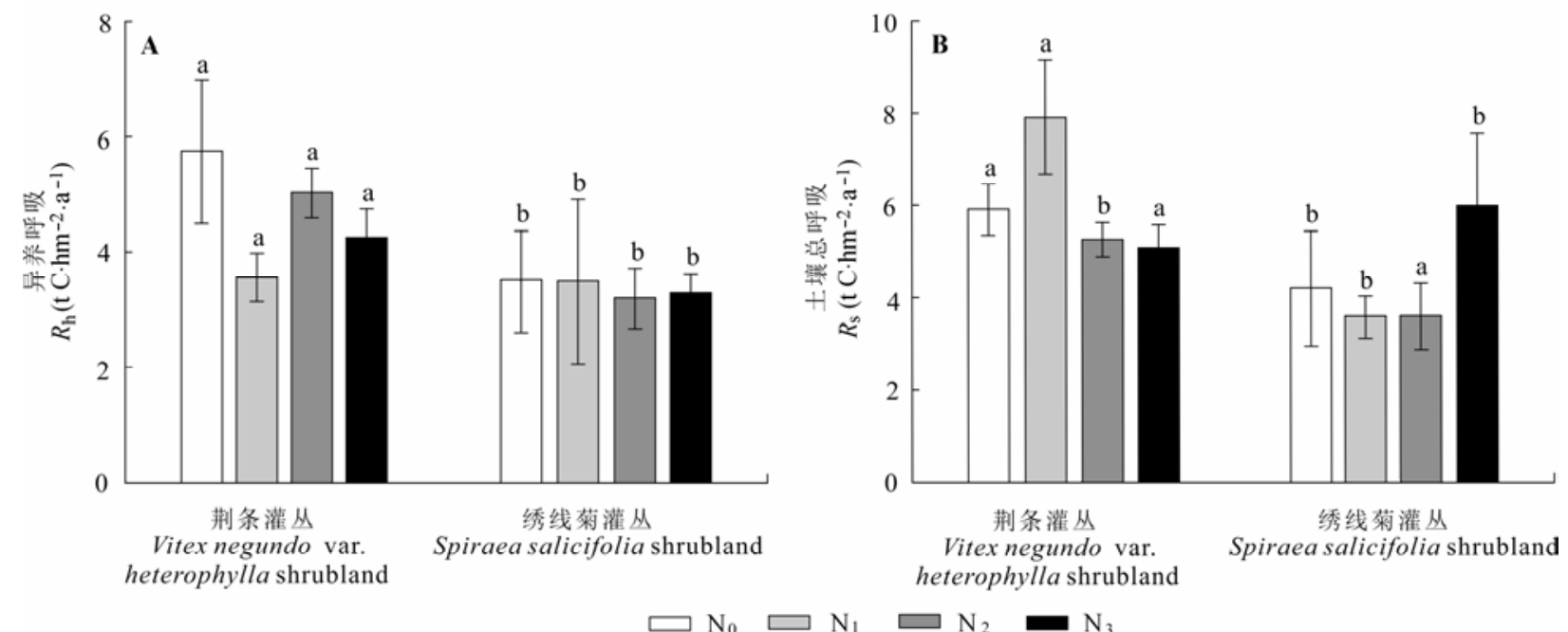

图7 氮添加对荆条和绣线菊灌从土壤异养呼吸 $\left(R_{\mathrm{h}}\right)$ 和土壤总呼吸 $\left(R_{\mathrm{s}}\right)$ 年通量的影响(平均值土标准误差)。相同字母 $\mathrm{a}$ 和 $\mathrm{b}$ 上标表 示各处理间无显著差异 $(p>0.05) 。 N_{0} 、 N_{1} 、 N_{2} 、 N_{3}$ 分别表示对照 $\left(0 \mathrm{~kg} \mathrm{~N} \cdot h m^{-2} \cdot a^{-1}\right)$ 、低氮 $\left(20 \mathrm{~kg} \mathrm{~N} \cdot h m^{-2} \cdot \mathrm{a}^{-1}\right) 、$ 中氮 $\left(50 \mathrm{~kg} \mathrm{~N} \cdot h m^{-2} \cdot \mathrm{a}^{-1}\right)$ 和高氮 $\left(100 \mathrm{~kg} \mathrm{~N} \cdot \mathrm{hm}^{-2} \cdot \mathrm{a}^{-1}\right)$ 处理。

Fig. 7 Influence of nitrogen addition on soil heterotrophic $\left(R_{\mathrm{h}}\right)$ and total $\left(R_{\mathrm{s}}\right)$ respiration in Vitex negundo var. heterophylla and Spiraea salicifolia shrublands (mean $\pm S E$ ). The same letter a and $b$ indicate no significant $(p>0.05)$ among treatments. $\mathrm{N}_{0}, \mathrm{~N}_{1}, \mathrm{~N}_{2}$ and $\mathrm{N}_{3}$ denote control $\left(0 \mathrm{~kg} \mathrm{~N} \cdot \mathrm{hm}^{-2} \cdot \mathrm{a}^{-1}\right)$, low $\left(20 \mathrm{~kg} \mathrm{~N} \cdot \mathrm{hm}^{-2} \cdot \mathrm{a}^{-1}\right)$, medium $\left(50 \mathrm{~kg} \mathrm{~N} \cdot \mathrm{hm}^{-2} \cdot \mathrm{a}^{-1}\right)$, and high $\left(100 \mathrm{~kg} \mathrm{~N} \cdot \mathrm{hm}^{-2} \cdot \mathrm{a}^{-1}\right)$ nitrogen addition, respectively. 


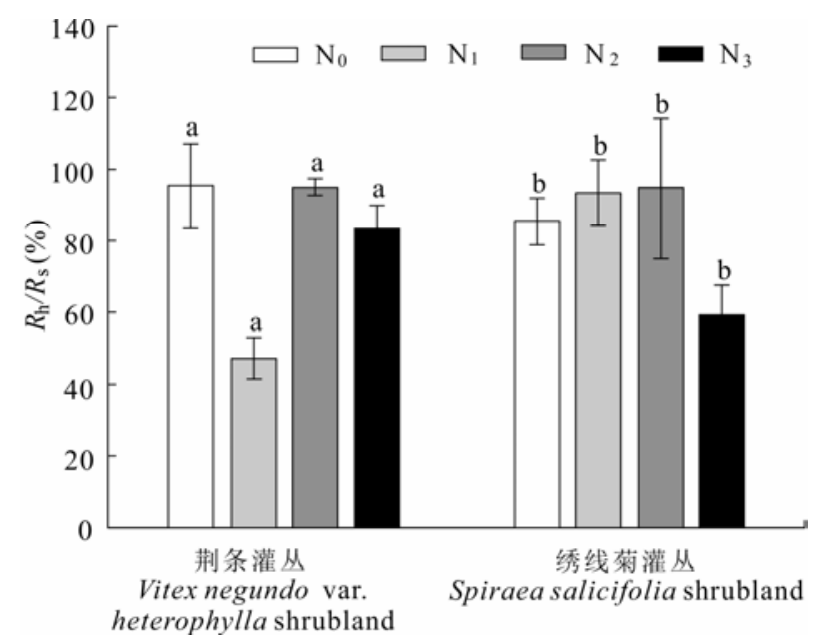

图8 氮添加对荆条和绣线菊灌从土壤异养呼吸组分贡献率 $\left(R_{\mathrm{h}} / R_{\mathrm{S}}\right)$ 的影响(平均值土标准误差)。图中相同字母表示各处理 间差异不显著 $(p>0.05) 。 N_{0} 、 N_{1} 、 N_{2} 、 N_{3}$ 分别表示对照 $(0 \mathrm{~kg}$ $\left.\mathrm{N} \cdot \mathrm{hm}^{-2} \cdot \mathrm{a}^{-1}\right)$ 、低氮 $\left(20 \mathrm{~kg} \mathrm{~N} \cdot \mathrm{hm}^{-2} \cdot \mathrm{a}^{-1}\right)$ 、中氮 $\left(50 \mathrm{~kg} \mathrm{~N} \cdot \mathrm{hm}^{-2} \cdot \mathrm{a}^{-1}\right)$ 和高氮 $\left(100 \mathrm{~kg} \mathrm{~N} \cdot \mathrm{hm}^{-2} \cdot \mathrm{a}^{-1}\right)$ 处理。

Fig. 8 Influence of nitrogen addition on contributions of soil heterotrophic respiration to total soil respiration $\left(R_{\mathrm{h}} / R_{\mathrm{s}}\right)$ in Vitex negundo var. heterophylla and Spiraea salicifolia shrublands (mean $\pm S E$ ). The same letter $\mathrm{a}$ and $\mathrm{b}$ indicate no significant $(p$ $>0.05$ ) among treatments. $\mathrm{N}_{0}, \mathrm{~N}_{1}, \mathrm{~N}_{2}$ and $\mathrm{N}_{3}$ denote control $\left(0 \mathrm{~kg} \mathrm{~N} \cdot \mathrm{hm}^{-2} \cdot \mathrm{a}^{-1}\right)$, low $\left(20 \mathrm{~kg} \mathrm{~N} \cdot \mathrm{hm}^{-2} \cdot \mathrm{a}^{-1}\right)$, medium (50 kg $\left.\mathrm{N} \cdot \mathrm{hm}^{-2} \cdot \mathrm{a}^{-1}\right)$, and high $\left(100 \mathrm{~kg} \mathrm{~N} \cdot \mathrm{hm}^{-2} \cdot \mathrm{a}^{-1}\right)$ nitrogen addition, respectively.

表4 2013年不同处理下土壤总呼吸和异养呼吸的年通量(平均值土标准 误差, $n=3$ )

Table 4 Annual flux of soil total $\left(R_{\mathrm{S}}\right)$ and heterotrophic $\left(R_{\mathrm{h}}\right)$ respiration (t $\mathrm{C} \cdot \mathrm{hm}^{-2} \cdot \mathrm{a}^{-1}$ ) in 2013 under different nitrogen addition treatments (mean \pm $S E, n=3)$

\begin{tabular}{lccc}
\hline & $\begin{array}{c}\text { 氮处理 } \\
\text { Nitrogen treatment }\end{array}$ & $\begin{array}{c}\text { 土壤总呼吸 } \\
R_{\mathrm{s}}\end{array}$ & $\begin{array}{c}\text { 异养呼吸 } \\
R_{\mathrm{h}}\end{array}$ \\
\hline 荆条灌从 & $\mathrm{N}_{0}$ & $5.91 \pm 0.54^{\mathrm{a}}$ & $5.76 \pm 1.24^{\mathrm{a}}$ \\
$\begin{array}{l}\text { Vitex negundo var. } \\
\text { heterophylla }\end{array}$ & $\mathrm{N}_{1}$ & $7.93 \pm 1.52^{\mathrm{a}}$ & $3.56 \pm 0.42^{\mathrm{a}}$ \\
shrubland & $\mathrm{N}_{2}$ & $5.30 \pm 0.36^{\mathrm{a}}$ & $5.04 \pm 0.42^{\mathrm{a}}$ \\
& $\mathrm{N}_{3}$ & $5.09 \pm 0.49^{\mathrm{a}}$ & $4.25 \pm 0.49^{\mathrm{a}}$ \\
绣线菊 & $\mathrm{N}_{0}$ & $4.23 \pm 1.23^{\mathrm{a}}$ & $3.53 \pm 0.88^{\mathrm{a}}$ \\
Spiraea salicifolia & $\mathrm{N}_{1}$ & $3.60 \pm 0.43^{\mathrm{a}}$ & $3.51 \pm 1.41^{\mathrm{a}}$ \\
shrubland & $\mathrm{N}_{2}$ & $3.60 \pm 0.71^{\mathrm{a}}$ & $3.21 \pm 0.51^{\mathrm{a}}$ \\
& $\mathrm{N}_{3}$ & $5.99 \pm 1.57^{\mathrm{a}}$ & $3.29 \pm 0.32^{\mathrm{a}}$ \\
\hline
\end{tabular}

表中相同字母表示各处理间差异不显著 $(p>0.05)$ 。 $\mathrm{N}_{0} 、 \mathrm{~N}_{1} 、 \mathrm{~N}_{2} 、 \mathrm{~N}_{3}$ 分 别表示对照 $\left(0 \mathrm{~kg} \mathrm{~N} \cdot \mathrm{hm}^{-2} \cdot \mathrm{a}^{-1}\right)$ 、低氮 $\left(20 \mathrm{~kg} \mathrm{~N} \cdot \mathrm{hm}^{-2} \cdot \mathrm{a}^{-1}\right)$ 、中氮 $(50 \mathrm{~kg}$ $\left.\mathrm{N} \cdot \mathrm{hm}^{-2} \cdot \mathrm{a}^{-1}\right)$ 和高氮 $\left(100 \mathrm{~kg} \mathrm{~N} \cdot \mathrm{hm}^{-2} \cdot \mathrm{a}^{-1}\right)$ 处理。

The same letter a and $\mathrm{b}$ indicate no significant $(p>0.05)$ among treatments. $\mathrm{N}_{0}, \mathrm{~N}_{1}, \mathrm{~N}_{2}$ and $\mathrm{N}_{3}$ denote control $\left(0 \mathrm{~kg} \mathrm{~N} \cdot \mathrm{hm}^{-2} \cdot \mathrm{a}^{-1}\right)$, low $\left(20 \mathrm{~kg} \mathrm{~N} \cdot \mathrm{hm}^{-2} \cdot \mathrm{a}^{-1}\right)$, medium $\left(50 \mathrm{~kg} \mathrm{~N} \cdot \mathrm{hm}^{-2} \cdot \mathrm{a}^{-1}\right)$, and high $\left(100 \mathrm{~kg} \mathrm{~N} \cdot \mathrm{hm}^{-2} \cdot \mathrm{a}^{-1}\right)$ nitrogen addition, respectively. $R_{\mathrm{h}}$, heterotrophic respiration; $R_{\mathrm{h}}$, total soil respiration.

大。说明不同植被类型土壤呼吸与纬度地带性的温 度变化有一定的相关性, 土壤呼吸的差异可能与气 候状况、植被类型、树龄和立地条件等因素有关(胡 正华等, 2010)。
土壤呼吸受到土壤温度、湿度等因子的综合影 响, 其过程十分复杂(彭勇等, 2015)。国内外许多研 究表明, 森林土壤呼吸与土壤温度有良好的相关性 (Jassal et al., 2007; 刘盛梅等, 2010)。在热带、亚热 带森林和北温带森林中均发现土壤呼吸与地表温度 之间具有极显著的指数关系(Jassal et al., 2007; 刘 盛梅等, 2010)。本研究利用温度单因素指数模型拟 合呼吸对温度的响应函数, 相关性极显著, 表明随 着温度的升高, 土壤呼吸呈指数增长, 与国内外许 多研究结果(Elberling \& Brandt, 2003; 陈宝玉等, 2007; 王小国等, 2007)相同。对众多植被的研究结 果表明, 土壤呼吸与土壤湿度之间也存在正相关关 系(Sotta et al., 2004)。本研究中土壤呼吸与土壤温度 和含水量表现出较强的相关性, 土壤含水量会影响 生物的代谢, 使得土壤水分成为土壤呼吸的限制因 子, 而温度通过直接影响植物、微生物活动和间接 地改变土壤湿度、输入土壤有机物的数量和质量对 土壤呼吸造成影响(Chapin, 2002), 成为东灵山灌从 土壤呼吸的另一主导影响因子。在各种生态系统中, $Q_{10}$ 在时间和空间上的变化可能非常大 $(\mathrm{Xu} \& \mathrm{Qi}$, 2001; Curiel et al., 2004), 本研究中 $Q_{10}$ 的值在1.4 2.1之间, 与全球 $Q_{10}$ 值范围(1.3-3.3) (Raich \& Potter, 1995)相一致。

本研究发现氮添加对荆条灌从生长季的土壤总 呼吸、异养呼吸无显著影响(图2; 表2)。这一结果与 长期实验所发现的施氮促进了土壤呼吸的结果 (Bowden et al., 2004; Micks et al., 2004; 莫江明等, 2005)并不一致。这可能与施肥时间短、实验期间降 水量少等因素导致土壤养分利用效率很低有关, 有 关机制还需要更长时间的施肥处理才能验证。 $\mathrm{N}_{2}$ 和 $\mathrm{N}_{3}$ 处理促进了绣线菊灌丛生长季土壤的异养呼吸, 这可能与氮添加导致绣线菊细根生物量和代谢强度 增加、增加土壤有机质进而增强微生物活性等有关 (Zak et al., 2000; Bowden et al., 2004; 涂利华等, 2010)。氮添加对荆条和绣线菊灌从土壤呼吸的年通 量均无显著的影响, 可能原因在于东灵山灌从地处 阳坡, 白天蒸发量大, 再加上在实验期间雨量较少, 导致灌从土壤含水量极低; 而土壤水分对土壤养分 的溶解转移、微生物活动、矿物分化等都有重要影 响, 土壤水分的缺乏导致上述过程受阻, 从而减弱 了施肥对呼吸的影响。研究发现, 氮添加会改变土 壤微生物群落结构, 而这种改变在不同类型森林会 
存在差异, 这会导致不同类型森林土壤呼吸对氮添 加的响应不同。此外, 氮素的添加效应还取决于氮 添加量、土壤氮素水平和土壤可利用性氮含量 (Demoling et al., 2008)。通常情况下, 低氮添加可以 促进植物生长、增加调落物产量, 使较多的碳分配 至地下部分等, 最终增强土壤呼吸(Bowden et al., 2004), 而高氮添加会导致土壤可利用性氮含量增 加, 引起植物地下部分的碳素分配减少(Litton et al., 2007), 最终抑制根际的自养呼吸 (Treseder, 2004)。氮添加还会降低微生物生物量和活性, 抑制 土壤有机质分解, 使土壤呼吸降低(Bowden et al., 2004)。

本研究同时发现, 氮添加对荆条和绣线菊灌从 土壤呼吸的温度敏感性均无显著影响, 可能是因为 施氮没有改变根系组织氮含量, 从而保持了较恒定 的细胞呼吸(贾淑霞等, 2007)。但两种灌从对氮添加 的响应存在差异, 具体表现在: $N_{1}$ 和 $N_{3}$ 在一定程度 上提高了荆条灌从土壤呼吸的 $Q_{10}$, 而 $\mathrm{N}_{2}$ 降低了其 土壤呼吸的 $Q_{10}$; 绣线菊灌从在相应氮处理下 $Q_{10}$ 的 变化趋势与荆条灌丛刚好相反, 这可能与氮添加改 变了荆条和绣线菊灌从土壤微生物数量和群落结构, 而不同的微生物类群对温度敏感性不同有关(彭勇 等, 2015)。此外, 与森林相比, 灌丛所处的环境异质 性较大, 样地用于测呼吸的Collar环所在的微环境可 能会非常不同, 会干扰氮添加的影响效应, 使短期 氮添加对灌从土壤呼吸 $Q_{10}$ 的影响趋势不明了。由于 施肥时间短, 氮沉降对土壤呼吸的影响可能存在阶 段性, 在长期持续氮沉降条件下, 灌丛土壤呼吸的 温度敏感性是如何变化的, 还需进行深入研究。

综上所述, 自然条件下, 北京东灵山灌从的土 壤呼吸主要受温度和土壤含水量的影响; 荆条和绣 线菊灌从土壤呼吸的年通量和土壤呼吸温度敏感性 对短期氮添加的响应均不显著, 但变化趋势不同, 可能与两灌从的群落组成结构和生态过程不同、试 验时间短、样地异质性等因素有关。灌从土壤呼吸 对未来持续增加的氮沉降会如何响应还有待进一步 研究。

基金项目 中国科学院战略先导性科技专项 (XDA05050300)和全球变化国家重大科学研究计划(2010CB950600和2014CB954004)。

\section{参考文献}

Allison SD, Czimczik CI, Treseser KK (2008). Microbial activ- ity and soil respiration under nitrogen addition in Alaskan boreal forest. Global Change Biology, 14, 1156-1168.

Ammann C, Flechard CR, Leifeld J, Neftel A, Fuhrer J (2007). The carbon budget of newly established temperate grassland depends on management intensity. Agriculture Ecosystems and Environment, 121, 5-20.

Bowden RD, Davidon E, Savage K, Arabia C, Steudler P (2004). Chronic nitrogen additions reduce total soil respiration and microbial respiration in temperate forest soils at the Harvard Forest. Forest Ecology and Management, 196, 43-56.

Bowden RD, Nadelhoffer KJ, Boone RD, Melillo JM, Garrison JB (1993). Contributions of aboveground litter, belowground litter, and root respiration to total soil respiration in a temperate mixed hardwood forest. Canadian Journal of Forest Research, 23, 1402-1407.

Bronson DR, Gower ST, Tanner M, Linder S, van Herk I (2008). Response of soil surface $\mathrm{CO}_{2}$ flux in a boreal forest to ecosystem warming. Global Change Biology, 14, 856-867.

Burton AJ, Pregitzer KS, Zogg GP, Zak DR (1998). Drought reduces root respiration in sugar maple forests. Ecological Applications, 8, 771-778.

Chapin III MC (2002). Principles of Terrestrial Ecosystem Ecology. Springer-Verlag, New York.

Chen BY, Liu SR, Ge JP, Wang H, Chang JG, Sun TT, Ma JM, Shi G (2007). The relationship between soil respiration and the temperature at different soil depths in subalpine coniferous forest of western Sichuan Province. Chinese Journal of Applied Ecology, 18, 1219-1224. (in Chinese with English abstract) [陈宝玉, 刘世荣, 葛剑平, 王辉, 常建国, 孙甜甜, 马姜明, 施恭 (2007). 川西亚高山针 叶林土壤呼吸速率与不同土层温度的关系. 应用生态 学报, 18, 1219-1224.]

Chen ST, Huang Y, Zou JW, Shen QR, Hu ZH, Qin YM, Chen HS, Pan GX (2010). Modeling interannual variability of global soil respiration from climate and soil properties. Agricultural and Forest Meteorology, 150, 590-605.

Cleveland CC, Townsend AR (2006). Nutrient additions to a tropical rain forest drive substantial soil carbon dioxide losses to the atmosphere. Proceedings of the National Academy of Sciences of the United States of America, 103, 10316-10321.

Curiel YJ, Janssens IA, Carrara A, Ceulemans R (2004). Annual $Q_{10}$ of soil respiration reflects plant phenological patterns as well as temperature sensitivity. Global Change Biology, 10, 161-169.

Davidson EA, Janssens IA (2006). Temperature sensitivity of soil carbon decomposition and feedbacks to climate change. Nature, 440, 165-173.

Demoling F, Nilsson LO, Baath E (2008). Bacterial and fungal response to nitrogen fertilization in three coniferous forest 
soils. Soil Biology \& Biochemistry, 40, 370-379.

Deng Q, Zhou GY, Liu JX, Liu SZ, Duan HL, Chen XM, Zhang DQ (2009). Effects of $\mathrm{CO}_{2}$ enrichment, high nitrogen deposition and high precipitation on a model forest ecosystem in southern China. Chinese of Journal of Plant Ecology, 33, 1023-1033. (in Chinese with English abstract) [邓琦, 周国逸, 刘菊秀, 刘世忠, 段洪浪, 陈小 梅, 张德强 (2009). $\mathrm{CO}_{2}$ 浓度倍增、高氮沉降和高降雨对 南亚热带人工模拟森林生态系统土壤呼吸的影响. 植 物生态学报, 33, 1023-1033.]

Diemer M (1997). Effects of elevated $\mathrm{CO}_{2}$ on gas exchange characteristics of alpine grassland. Acta Oecologica, 18, 177-182.

Du EZ (2013). Impacts of Nitrogen Enrichment on Carbon Cycling in an Old-growth Larch (Larix gmelinii Rupr.) Forest. PhD dissertation, Peking University, Beijing. (in Chinese with English abstract) [杜恩在 (2013). 氮添加 对兴安落叶松原始林碳收支主要过程的影响. 博士学 位论文, 北京大学, 北京.]

Du EZ, Zhou Z, Li P, Hu XY, Ma YC, Wang W, Zheng CY, Zhu JX, He JS, Fang JY (2013). NEECF: A project of nutrient enrichment experiments in China's forests. Journal of Plant Ecology, 6, 428-435.

Elberling B, Brandt KK (2003). Uncoupling of microbial $\mathrm{CO}_{2}$ production and release in frozen soil and its implications for field studies of arctic C cycling. Soil Biology \& Biochemistry, 35, 263-272.

Feng Y, Ma KM, Zhang YX, Qi J, Zhang JY (2007). Species abundance distribution of Quercus liaotungensis forest along altitudinal gradient in Dongling Mountain, Beijing. Acta Ecologica Sinica, 27, 4743-4750. (in Chinese with English abstract) [冯云, 马克明, 张育新, 祁建, 张洁瑜 (2007). 北京东灵山辽东栎(Quercus liaotungensis)林沿 海拔梯度的物种多度分布. 生态学报, 27, 4743-4750.]

Fisk MC, Fahey TJ (2001). Microbial biomass and nitrogen cycling responses to fertilization and litter removal in young northern hardwood forests. Biogeochemistry, 53, 201-223.

Galloway JN, Dentener FJ, Capone DG, Boyer EW, Howarth RW, Seitzinger SP, Asner GP, Clerveland CC, Green PA, Holland EA, Karl DM, Michaels AF, Porter JH, Townsend AR, Vörösmarty CJ (2004). Nitrogen cycles: Past, present, and future. Biogeochemistry, 70, 153-226.

Galloway JN, Townsend AR, Erisman JW, Bekunda M, Cai Z, Freney JR, Martinelli LA, Seitzinger SP, Sutton MA (2008). Transformation of the nitrogen cycle: Recent trends, questions, and potential solutions. Science, 20, 889-892.

Gorissen A, Tietema A, Joosten NN, Estiarte M, Penuelas J, Sowerby A, Bridget A, Emmett BA, Beier C (2004). Climate change affects carbon allocation to the soil in shrublands. Ecosystems, 7, 650-661.
Han Y, Zhang Z, Wang CH, Jiang FH, Xia JY (2012). Effects of mowing and nitrogen addition on soil respiration in three patches in an old field grassland in Inner Mongolia. Journal of Plant Ecology, 5, 219-228.

Hibbard KA, Law BE, Reichstein M, Sulzman J (2005). An analysis of soil respiration across northern hemisphere temperate ecosystems. Biogeochemistry, 73, 29-70.

Hu HF, Wang ZH, Liu GH, Fu BJ (2006). Vegetation carbon storage of major shrublands in China. Chinese of Journal of Plant Ecology (Chinese Version), 30, 539-544. (in Chinese with English abstract) [胡会峰, 王志恒, 刘国华, 傅 伯杰 (2006). 中国主要灌丛植被碳储量. 植物生态学 报, 30, 539-544.]

Hu SP, Yu XX, Guo YS (2010). Quantification analysis on site conditions of natural Vitex negundo community in Beijing mountainous area. Forest Resources Management, 3, 60-63. (in Chinese with English abstract) [胡淑萍, 余新 晓, 郭永盛 (2010). 北京山区天然荆条灌丛立地条件的 数量化分析. 林业资源管理, 3, 60-63.]

Hu ZH, Li HM, Yang YP, Chen ST, Li CZ, Shen SH (2010). Effects of simulated nitrogen deposition on soil respiration in northern subtropical deciduous broad-leaved forest. Environmental Science, 31, 1726-1732. (in Chinese with English abstract) [胡正华, 李涵茂, 杨燕萍, 陈书涛, 李 岑子, 申双和 (2010). 模拟氮沉降对北亚热带落叶阔叶 林土壤呼吸的影响. 环境科学, 31, 1726-1732.]

Jassal RS, Black TA, Cai T, Kai M, Li Z, Gaumont-Guay D, Nesic Z (2007). Components of ecosystem respiration and an estimate of net primary productivity of an Intermediateaged Douglas-fir stand. Agricultural and Forest Meteorology, 144, 44-57.

Jia SX, Wang ZQ, Mei L, Sun Y, Quan XK, Shi JW, Yu SQ, Sun HL, Gu JC (2007). Effect of nitrogen fertilization on soil respiration in Larix gmelinii and Fraxinus mandshurica plantations in China. Journal of Plant Ecology (Chinese Version), 31, 372-379. (in Chinese with English abstract) [贾淑霞, 王政权, 梅莉, 孙玥, 全先奎, 史建 伟, 于水强, 孙海龙, 谷加存 (2007). 施肥对落叶松和 水曲柳人工林土壤呼吸的影响. 植物生态学报, 31 , 372-379.]

Li DJ, Mo JM, Fang YT, Peng SL, Gundersen P (2003). Impact of nitrogen deposition on forest plants. Acta Ecologica Sinica, 3, 1891-1900. (in Chinese with English abstract) [李德军, 莫江明, 方运霆, 彭少麟, Gundersen P (2003). 氮沉降对森林植物的影响. 生态学报, 3, 1891-1900.]

Li QL, Xiao HL, Zeng XD, Feng YJ, Mo JM (2013). Effects of simulated nitrogen deposition on soil chemical properties of forests. Ecology and Environment Sciences, 22, 1872-1878. (in Chinese with English abstract) [李秋玲, 肖辉林, 曾晓舵, 冯乙晴, 莫江明 (2013). 模拟氮沉降 对森林土壤化学性质的影响. 生态环境学报, 22, 1872-

www.plant-ecology.com 
1878.]

Li WB, Jin CJ, Jing YL, Wu JB, Yuan FH, Guan DX, Wang AZ (2014). Response of soil respiration to enhanced nitrogen deposition in broadleaved Korean pine forest in Changbai Mountains. Journal of Northeast Forestry University, 12, 89-93. (in Chinese with English abstract) [李 伟斌, 金昌杰, 井艳丽, 吴家兵, 袁凤辉, 关德新, 王安 志 (2014). 长白山阔叶红松林土壤呼吸对氮沉降增加 的响应. 东北林业大学学报, 12, 89-93.]

Litton CM, Raich JW, Ryan MG (2007). Carbon allocation in forest ecosystems. Global Change Biology, 13, 20892109.

Liu CQ (1994). The study on techniques in determining shrub phytomass. Acta Pratacultural Science, 3, 61-65. (in Chinese with English abstract) [刘存琦 (1994). 灌木植物量 测定技术的研究. 草业学报, 3, 61-65.]

Liu SH, Fang JY (1997). Effect factors of soil respiration and the temperature's effects on soil respiration in the global scale. Acta Ecologica Sinica, 17, 469-476. (in Chinese with English abstract) [刘绍辉, 方精云 (1997). 土壤呼 吸的影响因素及全球尺度下温度的影响. 生态学报, 17 , 469-476.]

Liu SM, Jiang QC, Li Y (2010). Response of soil respiration to simulated nitrogen deposition under the middle-aged Eucalyptus grandis plantation in Ya'an City. Journal of Sichuan Forestry Science and Technology, 21, 60-64. (in Chinese with English abstract) [刘盛梅, 姜清成, 李芸 (2010). 华西雨屏区巨桉中龄林土壤呼吸对模拟氮沉降 的响应. 四川林业科技, 21, 60-64.]

Liu XR, Ren JQ, Li SG, Zhang QW (2015). Effects of simulated nitrogen deposition on soil net nitrogen mineralization in the meadow steppe of Inner Mongolia, China. PLOS ONE, 10, e0134039. doi: 10.1371/journal.pone.0134039.

Matson PA, Mcdowell WH, Townsend AR, Vitousek PM (1999). The globalization of $\mathrm{N}$ deposition: Ecosystem consequences in tropical environments. Biogeochemistry, 46, 67-83.

Micks P, Aber JD, Boone RD, Davidson EA (2004). Short term soil respiration and nitrogen immobilization response to nitrogen applications in control and nitrogen enriched temperate forests. Forest Ecology and Management, 196, 57-70.

Mo JM, Fang YT, Xu GL, Li DJ, Xue JH (2005). The shortterm responses of soil $\mathrm{CO}_{2}$ emission and $\mathrm{CH}_{4}$ uptake to simulated $\mathrm{N}$ deposition in nursery and forests of Dinghushan in subtropical China. Acta Ecologica Sinica, 25, 682-690. (in Chinese with English abstract) [莫江明, 方运霆, 徐国良, 李德军, 薛璟花 (2005). 鼎湖山苗圃 和主要森林土壤 $\mathrm{CO}_{2}$ 排放和 $\mathrm{CH}_{4}$ 吸收对模拟氮沉降的短 期响应. 生态学报, 25, 682-690.]

Peng Y, Chen G, Chen GT, Liang Z, Tu LH (2015). Effects of simulated nitrogen deposition on soil respiration in a secondary evergreen broad-leaved forest on Wawushan Mountain. Chinese Journal of Applied and Environmental Biology, 21, 733-739. (in Chinese with English abstract) [彭勇, 陈刚, 陈冠陶, 梁政, 涂利华 (2015). 模拟氮沉 降对瓦屋山常绿阔叶次生林土壤呼吸的影响. 应用与 环境生物学报, 21, 733-739.]

Qi YC, Liu XC, Dong YS, Peng Q, He YT, Sun LG, Jia JQ, Cao CC (2014). Differential responses of short-term soil respiration dynamics to the experimental addition of nitrogen and water in the temperate semi-arid steppe of Inner Mongolia, China. Journal of Environmental Sciences, 26, 834-845.

Quan Q, Zhang Z, He NP, Su HX, Wen XF, Sun XM (2015). Short-term effect of nitrogen addition on soil respiration of three temperate forests in Dongling Mountain. Chinese Journal of Ecology, 3, 797-804. (in Chinese with English abstract) [全权, 张震, 何念鹏, 苏宏新, 温学发, 孙晓 敏 (2015). 短期氮添加对东灵山三种森林土壤呼吸的 影响. 生态学杂志, 3, 797-804.]

Rabalais N (2002). Nitrogen in aquatic ecosystems. Ambio, 31, 102-112.

Raich JW, Polter CS (1995). Global patterns of carbon dioxide emissions from soils. Global Biogeochemistry Cycles, 9, 23-36.

Savage KE, Davidson EA (2001). Interannual variation of soil respiration in two New England forests. Global Biogeochemical Cycles, 15, 337-350.

Sitaula BK, Bakken LR, Abrahamsen G (1995). N-fertilization and soil acidification effects on $\mathrm{N}_{2} \mathrm{O}$ and $\mathrm{CO}_{2}$ emission from temperate pine forest soil. Soil Biology \& Biochemistry, 27, 1401-1408.

Sotta ED, Meir P, Malhi Y, Nobre AD, Hodnett M, Grace J (2004). Soil $\mathrm{CO}_{2}$ efflux in a tropical forest in the central Amazon. Global Change Biology, 10, 601-617.

Stevens CJ, Dise NB, Mountford JO, Gowing DJ (2004). Impact of nitrogen deposition on the species richness of grasslands. Science, 303, 1876-1879.

Treseder KK (2004). A meta-analysis of mycorrhizal responses to nitrogen, phosphorus, and atmospheric $\mathrm{CO}_{2}$ in field studies. New Phytologist, 164, 347-355.

Tu LH, Dai HZ, Hu TX, Zhang J, Luo SH (2011). Effects of simulated nitrogen deposition on soil respiration in a Bambusa pervariabilis $\times$ Dendrocala mopsi plantation in rainy area of West China. Chinese Journal of Applied Ecology, 22, 829-836. (in Chinese with English abstract) [涂利华, 戴洪忠, 胡庭兴, 张健, 雒守华 (2011). 模拟 氮沉降对华西雨屏区撑绿杂交竹林土壤呼吸的影响. 应用生态学报, 22, 829-836.]

Tu LH, Hu TX, Zhang J (2010). Effects of simulated nitrogen deposition on the fine root characteristics and soil respiration in a Pleioblastus amarus plantation in rainy area of 
West China. Chinese Journal of Applied Ecology, 21, 2472-2478. (in Chinese with English abstract) [涂利华, 胡庭兴, 张健 (2010). 模拟氮沉降对华西雨屏区苦竹林 细根特性和土壤呼吸的影响. 应用生态学报, 21, 2472-2478.]

Wang XG, Zhu B, Wang YQ, Zheng XH (2007). Soil respiration and its sensitivity to temperature under different land use conditions. Acta Ecologica Sinica, 27, 1960-1968. (in Chinese with English abstract) [王小国, 朱波, 王艳强, 郑循华 (2007). 不同土地利用方式下土壤呼吸及其温 度敏感性. 生态学报, 27, 1960-1968.]

Wessel WW, Tietema A, Beier C, Emmett BA, Peñuelas J, Nielsen TR (2004). A qualitative ecosystem assessment for different shrublands in western Europe under impact of climate change. Ecosystems, 7, 662-671.

Wu D, Zhang R, Gao SH, Fu X, Deng HB, Shao GF, Zhang XD (2015). Effects of simulated nitrogen deposition on the each component of soil respiration in the Populus L. plantations in a riparian zone of the mid-lower Yangtze River. Acta Ecologica Sinica, 35, 717-724. (in Chinese with English abstract) [吴迪, 张荵, 高升华, 付晓, 邓红兵, 邵国凡, 张旭东 (2015). 模拟氮沉降对长江中下游滩地 杨树林土壤呼吸各组分的影响. 生态学报, 35, 717724.]

Xie HH, Yang LL, Bao ZY (2006). Resources of the Spiraeas and their application to landscaping. Scientia Silvae Sinicae, 42(7), 104-112. (in Chinese with English abstract) [谢华辉, 杨莉莉, 包志毅 (2006). 绣线菊属植物资源 及其在园林中的应用前景. 林业科学, 42(7), 104-112.]

Xu M, Qi Y (2001). Spatial and seasonal variations of $Q_{10}$ determined by soil respiration measurements at a Sierra Nevada forest. Global Biogeochemical Cycles, 15, 687-696.

Yang QP, Xu M, Liu HS, Wang JS, Liu LX, Chi YG, Zheng YP (2011). Impact factors and uncertainties of the temperature sensitivity of soil respiration. Acta Ecologica Sinica, 31, 2301-2311. (in Chinese with English abstract) [杨庆朋, 徐明, 刘洪升, 王劲松, 刘丽香, 迟永刚, 郑 云普 (2011). 土壤呼吸温度敏感性的影响因素和不确 定性. 生态学报, 31, 2301-2311.]

Yao H, Hu XY, Zhu JL, Zhu JX, Ji CJ, Fang JY (2015). Soil respiration and the 20-year change in three temperate forests in Mt. Dongling, Beijing. Chinese Journal of Plant Ecology, 39, 849-856. (in Chinese with English abstract)
[姚辉, 胡雪洋, 朱江玲, 朱剑霄, 吉成均, 方精云 (2015). 北京东灵山3种温带森林土壤呼吸及其20年的 变化. 植物生态学报, 39, 849-856.]

Zak DR, Pregitzer KS, Curtis PS, Vogel CS, Holmes WE, Lussenhop J (2000). Atmospheric $\mathrm{CO}_{2}$, soil-N availability, and allocation of biomass and nitrogen by Populus tremuloides. Ecological Applications, 10, 34-46.

Zhang DQ, Shi PL, Zhang XZ (2005). Some advance in the main factors controlling soil respiration. Advances in Earth Science, 20, 778-785. (in Chinese with English abstract) [张东秋, 石培礼, 张宪洲 (2005). 土壤呼吸主要 影响因素的研究进展. 地球科学进展, 20, 778-785.]

Zhang JH (2015). Effects of Nitrogen Addition on Carbon Cycling of Shrublands in Mt. Dongling, Beijing. PhD dissertation, University of Chinese Academy of Sciences, Beijing. (in Chinese with English abstract) [张建华 (2015). 氮添加对北京东灵山灌从碳循环的影响. 博士学位论 文, 中国科学院大学, 北京.]

Zhang JR, Gao JR, Cui Q, Yang QL (2013). Point pattern analysis for relationships of Vitex negundo var. heterophylla in three typical stands. Journal of Zhejiang Agriculture and Forestry University, 30, 226-233. (in Chinese with English abstract) [张金瑞, 高甲荣, 崔强, 杨麒麟 (2013). 3种典型立地荆条种群及种间分布的空间点格 局. 浙江农林大学学报, 30, 226-233.]

Zhang Y, Hong M (2014). Response of soil respiration to experimental warming and nitrogen addition in Inner Mongolia desert steppe. Acta Agrestia Sinica, 22, 1227-1231. (in Chinese with English abstract) [张宇, 红梅 (2014). 内蒙古荒漠草原土壤呼吸对模拟增温和氮素添加的响 应. 草地学报, 22, 1227-1231.]

Zheng JJ, Fang HJ, Chen SL, Yu GR, Zhang PL, Xiu MJ, Zhang YN (2012). Effects of N addition on soil organic carbon components in an alpine meadow on the eastern Qinghai-Tibetan Plateau. Acta Ecologica Sinica, 32, 5363-5372. (in Chinese with English abstract) [郑娇娇, 方华军, 程淑兰, 于贵瑞, 张裴雷, 徐敏杰, 李英年 (2012). 增氮对青藏高原东缘典型高寒草甸土壤有机碳 组成的影响. 生态学报, 32, 5363-5372.]

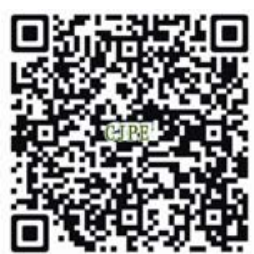

植物生态学报官网

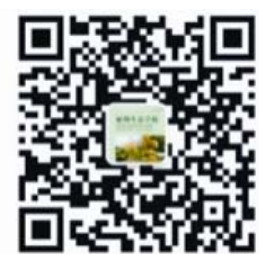

微信订阅号

期刊及学科

相关信息发布
责任编委: 谢宗强 责任编辑: 王 葳

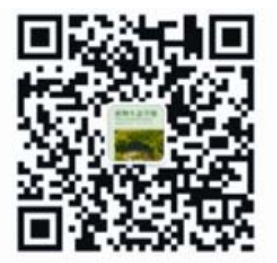

微信服务号

稿件状态查询

全文检索汶览 